\title{
Azerbaijan Republic: Statistical Appendix
}

This Statistical Appendix for the Azerbaijan Republic was prepared by a staff team of the International Monetary Fund as background documentation for the periodic consultation with the member country. It is based on the information available at the time it was completed on December 8 , 2004. The views expressed in this document are those of the staff team and do not necessarily reflect the views of the government of the Azerbaijan Republic or the Executive Board of the IMF.

The policy of publication of staff reports and other documents by the IMF allows for the deletion of market-sensitive information.

To assist the IMF in evaluating the publication policy, reader comments are invited and may be sent by e-mail to publicationpolicy@imf.org.

Copies of this report are available to the public from

International Monetary Fund • Publication Services

$70019^{\text {th }}$ Street, N.W. • Washington, D.C. 20431

Telephone: (202) 623-7430 • Telefax: (202) 623-7201

E-mail: publications@imf.org • Internet: http://www.imf.org

Price: $\$ 15.00$ a copy

\section{International Monetary Fund Washington, D.C.}





\section{INTERNATIONAL MONETARY FUND}

\section{AZERBAIJAN REPUBLIC}

\section{Statistical Appendix}

Prepared by a staff team comprising J. Wakeman-Linn, V. Kramarenko, N. Hobdari, K. Gvenetadze, (all MCD), E. Le Borgne (FAD), and B. Joshi (PDR) Approved by Middle East and Central Asia Department

December 8, 2004

Contents Page

Basic Data 2

1. Gross Domestic Product by Sector of Origin, 1999-2004 …………...........................

2. Gross Domestic Product by Final Use, 1999-2004 ...................................................

3. Income, Savings, and Net Financial Balances, 1999-2003 …………..........................

4. Crude Oil and Gas Production, 1981-2004 .............................................................6

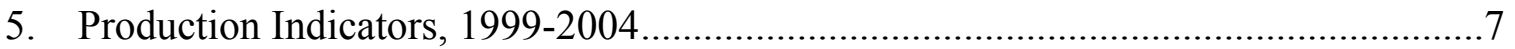

6. Average Monthly Wages by Sector, 1999-2004 .....................................................

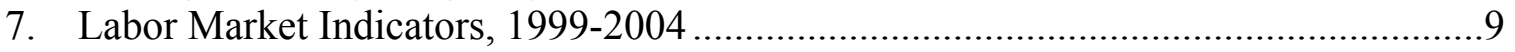

8. Consumer Price Index, 2000-04 .................................................................10

9. Breakdown of Consumer Price Index, 2000-04 ....................................................

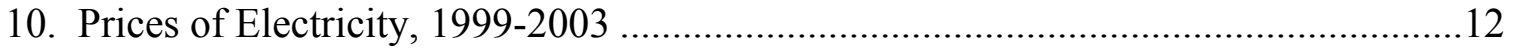

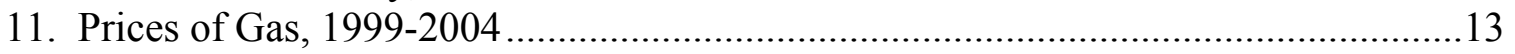

12. Consolidated Government Operations, 1999-2004...................................................14

13. Functional Classification of State Budget Expenditure, 1999-2004 ...........................15

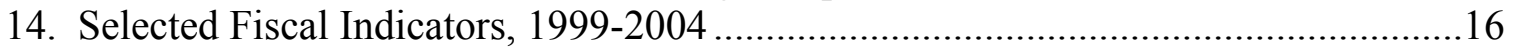

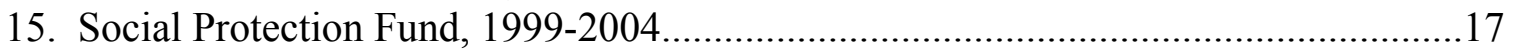

16. Summary Accounts of the Azerbaijan National Bank, 1999-2004...........................18

17. Summary Accounts of the Commercial Banks, 1999-2004......................................19

18. Net Bank Credit to the Consolidated Government, 1999-2004 .................................20

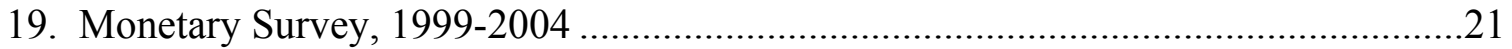

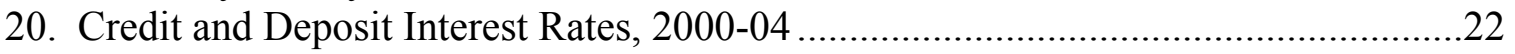

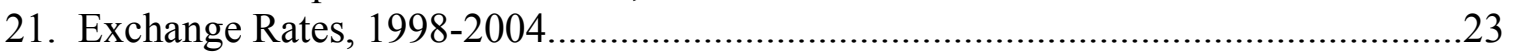

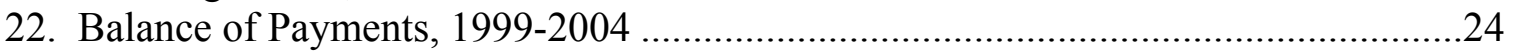

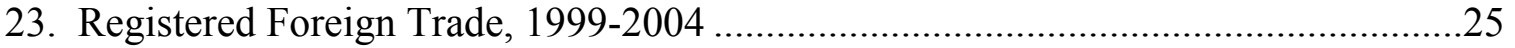

24. Balance of Services, Income and Transfers, 1999-2004..........................................26

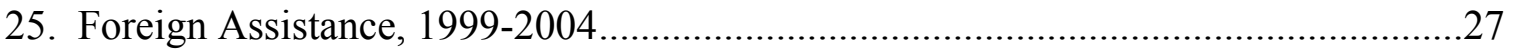

26. Direction of Registered Foreign Trade, 1999-2004 ..............................................28

27. Energy-Related Subsidies and Consolidated Government Operations, 2001-04 .......29

28. Energy Balance, 1999-2003 ...........................................................................

29. Collection Rates of Azerigas for Gas Supplied to Various Consumers, 1999-2004 ..31

30. Collection Rates of Azerenergy for Electricity Supplied to Various 
Azerbaijan: Basic Data

\begin{tabular}{|c|c|c|c|c|c|c|c|}
\hline \multicolumn{8}{|l|}{ Social and demographic indicators (2003) } \\
\hline Area (in sq. km) & \multirow{2}{*}{\multicolumn{7}{|c|}{$\begin{array}{r}86,600 \\
8,266.0\end{array}$}} \\
\hline Population (in thousands) & & & & & & & \\
\hline Percent urban & \multicolumn{7}{|c|}{51.0} \\
\hline Percent rural & \multicolumn{7}{|c|}{49.0} \\
\hline Population growth rate (in percent) & \multicolumn{7}{|c|}{0.8} \\
\hline Life expectancy at birth (in years) & \multicolumn{7}{|c|}{$\begin{array}{l}0.8 \\
72\end{array}$} \\
\hline Infant mortality rate (per 1,000 population) & \multicolumn{7}{|c|}{12.8} \\
\hline Physicians (per 1,000 population) & \multicolumn{7}{|c|}{3.6} \\
\hline \multirow{3}{*}{ GDP per capita (in U.S. dollars, at average 2003 exchange rate) } & \multicolumn{7}{|c|}{862.5} \\
\hline & \multirow[t]{2}{*}{1999} & \multirow[t]{2}{*}{2000} & \multirow[t]{2}{*}{2001} & 2002 & \multicolumn{2}{|c|}{2003} & 2004 \\
\hline & & & & & First half & $\begin{array}{c}\text { Year } \\
\end{array}$ & First half \\
\hline & \multicolumn{7}{|c|}{ (In percent of GDP) } \\
\hline \multicolumn{8}{|l|}{ Structure of GDP } \\
\hline Industry and construction & 39.1 & 42.5 & 43.5 & 46.2 & 51.1 & 49.9 & 53.3 \\
\hline of which: Oil and gas sector & 20.1 & 30.5 & 32.0 & 31.0 & 33.7 & 31.1 & 32.6 \\
\hline Agriculture & 18.2 & 15.9 & 14.8 & 14.0 & 4.3 & 13.1 & 4.1 \\
\hline Other & 42.7 & 41.5 & 41.7 & 39.9 & 44.6 & 36.9 & 42.6 \\
\hline & \multicolumn{7}{|c|}{ (Annual percentage change, unless otherwise indicated) } \\
\hline Output and employment & & & & & & & \\
\hline GDP (in billions of manats) & 18,875 & 23,591 & 26,578 & 30,312 & 16,171 & 35,054 & 18,520 \\
\hline Real GDP & 7.9 & 10.3 & 9.6 & 9.7 & 10.1 & 10.8 & 10.6 \\
\hline Employment & 0.1 & -0.1 & 0.3 & 0.3 & $\ldots$ & 0.5 & 0.4 \\
\hline \multicolumn{8}{|l|}{ Prices and wages } \\
\hline \multicolumn{8}{|l|}{ Consumer price inflation } \\
\hline Average & -8.5 & 1.8 & 1.5 & 2.8 & 2.7 & 2.2 & 4.2 \\
\hline End of Period & -0.5 & 2.2 & 1.3 & 3.3 & 1.9 & 3.6 & 5.5 \\
\hline Average nominal manat wage & 9.5 & 20.2 & 17.3 & 21.2 & $\ldots$ & 22.8 & 19.4 \\
\hline Real manat wage & 19.6 & 18.1 & 15.5 & 18.0 & $\ldots$ & 20.1 & 14.6 \\
\hline Average nominal dollar wage & 2.8 & 10.7 & 12.7 & 16.1 & $\ldots$ & 21.5 & 19.0 \\
\hline Real dollar wage & 12.3 & 8.7 & 11.0 & 13.0 & $\ldots$ & 18.9 & 14.2 \\
\hline & & (In mill & s of U.S. & llars, unles & ss otherwise ind & cated) & \\
\hline External sector & & & & & & & \\
\hline Exports of goods & 1,025 & 1,799 & 2,046 & 2,305 & 1,254 & 2,625 & 1,679 \\
\hline Imports of goods & 1,433 & 1,539 & 1,465 & 1,823 & 1,164 & 2,723 & 1,553 \\
\hline Current account balance & -600 & -187 & -50 & -769 & -800 & $-2,021$ & $-1,266$ \\
\hline (in percent of GDP) & -13.1 & -3.6 & -0.9 & -12.3 & -24.3 & -28.3 & -33.6 \\
\hline & & & & ercent of $\mathrm{C}$ & GDP) & & \\
\hline Consolidated Government & & & & & & & \\
\hline Total revenue $/ 1$ & 18.5 & 21.2 & 18.7 & 27.3 & 27.7 & 27.1 & 28.6 \\
\hline Total expenditure (including net lending) $1 /$ & 23.6 & 20.8 & 18.7 & 27.7 & 29.9 & 28.9 & 26.9 \\
\hline Fiscal balance (- deficit) $2 /$ & -4.7 & -0.6 & -0.4 & -0.5 & -1.7 & -1.2 & 2.4 \\
\hline & & (Annua & rcentage & ange, unles & ss otherwise in & cated) & \\
\hline Financial markets & & & & & & & \\
\hline Manat reserve money, end of period & 7.0 & 18.2 & 9.4 & 11.1 & $19.83 /$ & 23.7 & 38.83 \\
\hline Manat broad money, end of period & 5.3 & 11.1 & 7.7 & 15.5 & $31.63 /$ & 28.0 & 30.93 \\
\hline Manat velocity relative to non-oil GDP (ratio) 4/ & 10.6 & 11.4 & 11.3 & 11.6 & $\ldots$ & 10.9 & $\ldots$ \\
\hline Exchange rate (manat/US\$) & & & & & & & \\
\hline End of period & 4,378 & 4,565 & 4,775 & 4,893 & $4,9133 /$ & 4,923 & 4,9053 \\
\hline Period average & 4,120 & 4,474 & 4,657 & 4,861 & $4,9073 /$ & 4,911 & 4,9233 \\
\hline
\end{tabular}

Sources: Azerbaijan State Statistics Committee, Azerbaijan National Bank, Ministry of Finance; and Fund staff estimates.

1/ Starting from 2002 revenue and expenditure include tax credits to SOCAR for energy-related subsidies.

2/ Calculated from below the line data.

3/ For nine months.

4/ Defined as non-oil GDP divided by average manat broad money. 
Table 1. Azerbaijan: Gross Domestic Product by Sector of Origin, 1999-2004

\begin{tabular}{|c|c|c|c|c|c|c|c|}
\hline & \multirow[t]{2}{*}{1999} & \multirow[t]{2}{*}{2000} & \multirow[t]{2}{*}{2001} & \multirow[t]{2}{*}{2002} & \multicolumn{2}{|c|}{2003} & \multirow{2}{*}{$\begin{array}{c}2004 \\
\text { First half }\end{array}$} \\
\hline & & & & & First half & Year & \\
\hline & \multicolumn{7}{|c|}{ (In billions of manats) } \\
\hline Gross Domestic Product at market prices & 18,875 & 23,591 & 26,578 & 30,312 & 16,171 & 35,054 & 18,520 \\
\hline Taxes on goods and services & 1,336 & 1,512 & 2,166 & 2,431 & 1,219 & 2,439 & 1,282 \\
\hline Subsidies on goods and services & 590 & 56 & 74 & 0 & 0 & 0 & 0 \\
\hline Gross Domestic Product at factor cost & 18,129 & 22,135 & 24,486 & 27,881 & 14,952 & 32,614 & 17,238 \\
\hline Industry & 5,327 & 8,495 & 9,999 & 11,350 & 6,628 & 13,252 & 7,390 \\
\hline Extraction & 3,493 & 6,522 & 7,880 & 8,756 & 5,035 & 10,033 & 5,548 \\
\hline Of which: oil and gas extraction & 3,477 & 6,511 & 7,875 & 8,744 & 5,029 & 10,019 & 5,539 \\
\hline Processing & 1,140 & 1,248 & 1,731 & 2,253 & 1,384 & 2,819 & 1,625 \\
\hline Of which: oil and gas processing & 316 & 676 & 632 & 667 & 415 & 896 & 490 \\
\hline Electricity, gas and water supply & 694 & 725 & 387 & 341 & 209 & 400 & 217 \\
\hline Agriculture & 3,440 & 3,755 & 3,943 & 4,230 & 702 & 4,605 & 763 \\
\hline Construction & 2,053 & 1,540 & 1,554 & 2,640 & 1,632 & 4,250 & 2,481 \\
\hline Transportation and communication & 2,022 & 2,836 & 2,695 & 2,985 & 1,680 & 3,333 & 1,864 \\
\hline Trade & 1,336 & 1,575 & 1,966 & 2,267 & 1,328 & 2,582 & 1,593 \\
\hline Social services & 3,951 & 3,934 & 4,330 & 4,409 & 2,982 & 4,592 & 3,147 \\
\hline
\end{tabular}

Source: Azerbaijan State Statistics Committee. 
Table 2. Azerbaijan: Gross Domestic Product by Final Use, 1999-2004

\begin{tabular}{|c|c|c|c|c|c|c|c|}
\hline & \multirow[t]{2}{*}{1999} & \multirow[t]{2}{*}{2000} & \multirow[t]{2}{*}{2001} & \multirow[t]{2}{*}{2002} & \multicolumn{2}{|c|}{2003} & \multirow{2}{*}{$\begin{array}{c}2004 \\
\text { First half }\end{array}$} \\
\hline & & & & & First half & Year & \\
\hline & \multicolumn{7}{|c|}{ (In billions of manats) } \\
\hline Gross Domestic Product & 18,875 & 23,591 & 26,578 & 30,312 & 16,171 & 35,054 & 18,520 \\
\hline Final consumption & 16,497 & 18,556 & 20,125 & 22,781 & 11,505 & 25,521 & 13,327 \\
\hline Households & 13,656 & 15,371 & 17,102 & 18,477 & 10,150 & 22,240 & 11,000 \\
\hline Government & 2,840 & 3,185 & 3,023 & 4,304 & 1,355 & 3,281 & 2,327 \\
\hline Gross fixed capital formation & 5,381 & 5,459 & 6,081 & 10,330 & 7,199 & 17,810 & 11,609 \\
\hline Private & 4,688 & 4,746 & 5,534 & 8,631 & 6,263 & 15,667 & 10,100 \\
\hline Public & 693 & 713 & 547 & 1,699 & 936 & 2,142 & 1,509 \\
\hline Change in inventories & -380 & -581 & -586 & -592 & -55 & 133 & 0 \\
\hline Resource gap & $-2,623$ & 156 & 958 & $-2,207$ & $-2,773$ & $-8,409$ & $-6,225$ \\
\hline Exports of goods and nonfactor services & 5,282 & 9,210 & 10,877 & 12,964 & 7,646 & 15,006 & 8,271 \\
\hline Imports of goods and nonfactor services & 7,905 & 9,054 & 9,918 & 15,171 & 10,419 & 23,415 & 14,496 \\
\hline \multirow[t]{2}{*}{ Statistical discrepancy } & 0 & 0 & 0 & 0 & 295 & 0 & -191 \\
\hline & \multicolumn{7}{|c|}{ (In percent of GDP) } \\
\hline Gross Domestic Product & 100.0 & 100.0 & 100.0 & 100.0 & 100.0 & 100.0 & 100.0 \\
\hline Final consumption & 87.4 & 78.7 & 75.7 & 75.2 & 71.1 & 72.8 & 72.0 \\
\hline Households & 72.4 & 65.2 & 64.3 & 61.0 & 62.8 & 63.4 & 59.4 \\
\hline Government & 15.0 & 13.5 & 11.4 & 14.2 & 8.4 & 9.4 & 12.6 \\
\hline Gross fixed capital formation & 28.5 & 23.1 & 22.9 & 34.1 & 44.5 & 50.8 & 62.7 \\
\hline Private & 24.8 & 20.1 & 20.8 & 28.5 & 38.7 & 44.7 & 54.5 \\
\hline Public & 3.7 & 3.0 & 2.1 & 5.6 & 5.8 & 6.1 & 8.1 \\
\hline Change in inventories & -2.0 & -2.5 & -2.2 & -2.0 & -0.3 & 0.4 & 0.0 \\
\hline Net exports & -13.9 & 0.7 & 3.6 & -7.3 & -17.1 & -24.0 & -33.6 \\
\hline Exports of goods and nonfactor services & 28.0 & 39.0 & 40.9 & 42.8 & 47.3 & 42.8 & 44.7 \\
\hline Imports of goods and nonfactor services & 41.9 & 38.4 & 37.3 & 50.0 & 64.4 & 66.8 & 78.3 \\
\hline Statistical discrepancy & 0.0 & 0.0 & 0.0 & 0.0 & 1.8 & 0.0 & -1.0 \\
\hline
\end{tabular}

Sources: Azerbaijan State Statistics Committee; and Fund staff estimates. 
Table 3. Azerbaijan: Income, Savings, and Net Financial Balances, 1999-2003

\begin{tabular}{|c|c|c|c|c|c|}
\hline & 1999 & 2000 & 2001 & 2002 & 2003 \\
\hline & \multicolumn{5}{|c|}{ (In billions of manats) } \\
\hline GDP at market prices & 18,875 & 23,591 & 26,578 & 30,312 & 35,054 \\
\hline Net factor income from abroad & 191 & $-1,321$ & $-1,547$ & $-1,874$ & $-2,172$ \\
\hline Gross national product & 19,066 & 22,269 & 25,031 & 28,438 & 32,881 \\
\hline Unrequited transfers (net) & 357 & 327 & 357 & 342 & 613 \\
\hline Gross disposable national income & 19,423 & 22,596 & 25,387 & 28,780 & 33,494 \\
\hline Total consumption & 16,497 & 18,556 & 20,125 & 22,781 & 25,521 \\
\hline Private & 13,656 & 15,371 & 17,102 & 18,477 & 22,240 \\
\hline General government & 2,840 & 3,185 & 3,023 & 4,304 & 3,281 \\
\hline Gross national savings $1 /$ & 2,926 & 4,040 & 5,262 & 5,999 & 7,973 \\
\hline Private & 3,128 & 3,464 & 4,830 & 4,445 & 6,256 \\
\hline General government & -202 & 576 & 433 & 1,555 & 1,718 \\
\hline Gross domestic savings 2/ & 2,378 & 5,034 & 6,453 & 7,531 & 9,533 \\
\hline Gross fixed investment & 5,381 & 5,459 & 6,081 & 10,330 & 17,810 \\
\hline Private sector & 4,688 & 4,746 & 5,534 & 8,631 & 15,667 \\
\hline \multirow[t]{2}{*}{ General government } & 693 & 713 & 547 & 1,699 & 2,142 \\
\hline & \multicolumn{5}{|c|}{ (In percent of GDP) } \\
\hline GDP at market prices & 100.0 & 100.0 & 100.0 & 100.0 & 100.0 \\
\hline Net factor income from abroad & 1.0 & -5.6 & -5.8 & -6.2 & -6.2 \\
\hline Gross national product & 101.0 & 94.4 & 94.2 & 93.8 & 93.8 \\
\hline Unrequited transfers (net) & 1.9 & 1.4 & 1.3 & 1.1 & 1.7 \\
\hline Gross disposable national income & 102.9 & 95.8 & 95.5 & 94.9 & 95.6 \\
\hline Total consumption & 87.4 & 78.7 & 75.7 & 75.2 & 72.8 \\
\hline Private & 72.4 & 65.2 & 64.3 & 61.0 & 63.4 \\
\hline General government & 15.0 & 13.5 & 11.4 & 14.2 & 9.4 \\
\hline Gross national savings $1 /$ & 15.5 & 17.1 & 19.8 & 19.8 & 22.7 \\
\hline Private & 16.6 & 14.7 & 18.2 & 14.7 & 17.8 \\
\hline General government & -1.1 & 2.4 & 1.6 & 5.1 & 4.9 \\
\hline Gross domestic savings 2/ & 12.6 & 21.3 & 24.3 & 24.8 & 27.2 \\
\hline Gross fixed investment & 28.5 & 23.1 & 22.9 & 34.1 & 50.8 \\
\hline Private sector & 24.8 & 20.1 & 20.8 & 28.5 & 44.7 \\
\hline General government & 3.7 & 3.0 & 2.1 & 5.6 & 6.1 \\
\hline
\end{tabular}

Sources: Azeri authorities; and Fund staff estimates.

1/ Gross disposable national income minus total consumption.

2/ GDP at market prices minus total consumption. 
Table 4. Azerbaijan: Crude Oil and Gas Production, 1981-2004

\begin{tabular}{|c|c|c|c|c|c|}
\hline & \multicolumn{2}{|c|}{ Offshore crude oil } & \multirow{2}{*}{$\begin{array}{l}\text { Onshore } \\
\text { crude oil }\end{array}$} & \multirow{2}{*}{$\begin{array}{r}\text { Total } \\
\text { crude oil }\end{array}$} & \multirow{2}{*}{$\begin{array}{r}\text { Total } \\
\text { gas }\end{array}$} \\
\hline & Total & $\begin{array}{r}\text { Of which: } \\
\text { AIOC 1/ }\end{array}$ & & & \\
\hline & \multicolumn{3}{|c|}{ (In millions of tons) } & \multicolumn{2}{|c|}{$\begin{array}{l}\text { In billions of ) } \\
\text { cubic meters) }\end{array}$} \\
\hline 1981 & 9.4 & - & 4.7 & 14.1 & 14.6 \\
\hline 1982 & 8.2 & - & 4.7 & 12.9 & 14.9 \\
\hline 1983 & 8.4 & - & 4.3 & 12.7 & 14.5 \\
\hline 1984 & 8.4 & - & 4.1 & 12.5 & 14.4 \\
\hline 1985 & 9.2 & - & 3.9 & 13.1 & 14.1 \\
\hline 1986 & 9.4 & - & 3.9 & 13.3 & 13.6 \\
\hline 1987 & 10.1 & - & 3.7 & 13.8 & 12.5 \\
\hline 1988 & 10.3 & - & 3.4 & 13.7 & 11.8 \\
\hline 1989 & 10.2 & - & 3.0 & 13.2 & 11.1 \\
\hline 1990 & 9.9 & - & 2.6 & 12.5 & 9.9 \\
\hline 1991 & 9.5 & - & 2.2 & 11.7 & 8.6 \\
\hline 1992 & 9.1 & - & 2.0 & 11.1 & 7.9 \\
\hline 1993 & 8.3 & - & 2.0 & 10.3 & 6.8 \\
\hline 1994 & 7.8 & - & 1.8 & 9.6 & 6.4 \\
\hline 1995 & 7.5 & - & 1.6 & 9.1 & 6.6 \\
\hline 1996 & 7.5 & - & 1.6 & 9.1 & 6.3 \\
\hline 1997 & 7.5 & 0.1 & 1.6 & 9.1 & 6.0 \\
\hline 1998 & 9.8 & 2.4 & 1.6 & 11.4 & 5.6 \\
\hline 1999 & 12.3 & 4.8 & 1.5 & 13.8 & 6.0 \\
\hline 2000 & 12.5 & 5.0 & 1.5 & 14.0 & 6.0 \\
\hline 2001 & 13.3 & 5.9 & 1.6 & 14.9 & 5.5 \\
\hline 2002 & 13.8 & 6.5 & 1.5 & 15.3 & 5.1 \\
\hline 2003 & 13.8 & 6.5 & 1.6 & 15.4 & 5.1 \\
\hline \multicolumn{6}{|l|}{ Of which: } \\
\hline First half of 2003 & 6.1 & 3.2 & 1.6 & 7.7 & 2.5 \\
\hline 2004 (first half) & 6.8 & 4.7 & 0.9 & 7.7 & 2.5 \\
\hline
\end{tabular}

Sources: Azerbaijan State Statistics Committee.

1/ Azerbaijan International Oil Company. 
Table 5. Azerbaijan: Production Indicators, 1999-2004

\begin{tabular}{|c|c|c|c|c|c|c|c|}
\hline & \multirow[t]{2}{*}{1999} & \multirow[t]{2}{*}{2000} & \multirow[t]{2}{*}{2001} & \multirow[t]{2}{*}{2002} & \multicolumn{2}{|c|}{2003} & \multirow{2}{*}{$\frac{2004}{\text { First half }}$} \\
\hline & & & & & First half & Year & \\
\hline & \multicolumn{7}{|c|}{ (Index of real output; $1992=100$ ) } \\
\hline Total industry & 47.1 & 50.3 & 52.9 & 54.8 & 58.2 & 58.1 & 65.2 \\
\hline Energy & 82.4 & 83.9 & 85.2 & 87.1 & 90.4 & 89.2 & 91.3 \\
\hline Electricity & 75.8 & 78.2 & 79.7 & 78.5 & 89.1 & 88.4 & 89.6 \\
\hline Oil and gas & 85.7 & 90.2 & 91.6 & 93.7 & 96.3 & 94.4 & 96.6 \\
\hline Metallurgy & 7.1 & 17.8 & 7.4 & 14.5 & 26.7 & 26.0 & 38.7 \\
\hline Machine building & 6.3 & 12.3 & 14.7 & 12.0 & 13.6 & 25.2 & 43.6 \\
\hline Chemical and petro-chemical & 32.2 & 39.7 & 22.9 & 29.8 & 29.8 & 32.6 & 38.7 \\
\hline Construction materials & 9.5 & 11.4 & 21.0 & 29.8 & 39.2 & 35.8 & 53.3 \\
\hline Light industry & 9.3 & 11.5 & 8.9 & 9.6 & 8.6 & 9.7 & 11.8 \\
\hline Textiles & 10.0 & 12.3 & 9.7 & 12.2 & 10.9 & 14.2 & 20.0 \\
\hline Agro processing & 15.9 & 16.2 & 18.4 & 19.9 & 18.4 & 20.9 & 21.0 \\
\hline Agriculture 1/ & 75.4 & 84.5 & 93.9 & 99.9 & $\ldots$ & 105.5 & $\ldots$ \\
\hline Grains & 82.1 & 115.2 & 150.8 & 164.2 & $\ldots$ & 153.9 & $\ldots$ \\
\hline \multirow[t]{2}{*}{ Cotton } & 28.8 & 27.2 & 24.9 & 23.9 & $\ldots$ & 29.6 & $\ldots$ \\
\hline & \multicolumn{7}{|c|}{ (Percentage changes) } \\
\hline Total industry & 3.5 & 6.8 & 5.2 & 3.6 & -- & 6.0 & 12.0 \\
\hline Energy & 5.2 & 1.8 & 1.5 & 2.2 & -- & 2.4 & 1.0 \\
\hline Electricity & 1.5 & 3.2 & 1.9 & -1.5 & -- & 12.6 & 0.6 \\
\hline Oil and gas & 7.0 & 5.3 & 1.6 & 2.3 & -- & 0.7 & 0.3 \\
\hline Metallurgy & 61.4 & 150.7 & -58.4 & 95.9 & -- & 79.3 & 44.9 \\
\hline Machine building & -55.6 & 95.2 & 19.5 & -18.4 & -- & 110.0 & 220.6 \\
\hline Chemical and petro-chemical & 8.8 & 23.3 & -42.3 & 30.1 & -- & 9.4 & 29.9 \\
\hline Construction materials & -21.5 & 20.0 & 84.2 & 41.9 & -- & 20.1 & 36.0 \\
\hline Light industry & -40.4 & 23.7 & -22.6 & 7.9 & -- & 1.0 & 37.2 \\
\hline Textiles & -34.6 & 23.0 & -21.1 & 25.8 & -- & 16.4 & 83.5 \\
\hline Agro processing & 3.9 & 1.9 & 13.6 & 8.2 & -- & 5.0 & 14.1 \\
\hline Agriculture 1/ & 7.0 & 12.1 & 11.1 & 6.4 & -- & 5.6 & $\ldots$ \\
\hline Grains & 15.5 & 40.3 & 30.9 & 8.9 & -- & -6.3 & $\ldots$ \\
\hline Cotton & -14.3 & -5.6 & -8.5 & -4.0 & -- & 23.8 & $\ldots$ \\
\hline
\end{tabular}

Source: Azerbaijan State Statistics Committee.

1/ Given the seasonality factor in agricultural production, the State Statistics Committee does not provide data for comparison of production on a half-year basis. 
Table 6. Azerbaijan: Average Monthly Wages by Sector, 1999-2004

\begin{tabular}{|c|c|c|c|c|c|c|c|}
\hline & \multirow[t]{2}{*}{1999} & \multirow[t]{2}{*}{2000} & \multirow[t]{2}{*}{2001} & \multirow[t]{2}{*}{2002} & \multicolumn{2}{|c|}{2003} & \multirow{2}{*}{$\frac{2004}{\text { First half }}$} \\
\hline & & & & & First half & Year & \\
\hline & \multicolumn{6}{|c|}{ (Annual average, in manats) } & \\
\hline National economy & 184,368 & 221,606 & 259,991 & 315,219 & 373,537 & 386,974 & 445,842 \\
\hline Industry & 365,575 & 436,237 & 499,763 & 554,398 & 638,955 & 686,405 & 893,615 \\
\hline Agriculture & 65,122 & 69,081 & 78,936 & 88,729 & 86,277 & 115,692 & 128,684 \\
\hline Transportation and communication & 249,870 & 292,421 & 330,281 & 365,414 & 414,644 & 439,961 & 531,496 \\
\hline Construction & 428,473 & 416,663 & 436,924 & 548,286 & 779,596 & 771,948 & $1,042,348$ \\
\hline Trade & 117,943 & 119,284 & 149,648 & 175,115 & 441,708 & 484,681 & 571,843 \\
\hline Hotel industry & 343,351 & 314,858 & 419,447 & 427,140 & 389,820 & 508,525 & 512,760 \\
\hline Health and social services & 71,098 & 73,395 & 80,630 & 89,949 & 90,179 & 109,516 & 125,377 \\
\hline Education & 141,532 & 156,065 & 165,225 & 168,961 & 178,944 & 211,396 & 243,514 \\
\hline Banking and insurance & 590,961 & 632,626 & 723,606 & $1,019,767$ & 930,265 & 979,801 & $1,035,952$ \\
\hline \multirow[t]{2}{*}{ Government administration } & 181,746 & 194,528 & 223,421 & 260,356 & 276,601 & 314,921 & 347,963 \\
\hline & \multicolumn{6}{|c|}{ (Annual percentage change) } & \\
\hline National economy & 9.5 & 20.2 & 17.3 & 21.2 & -- & 22.8 & 19.4 \\
\hline Industry & 32.0 & 19.3 & 14.6 & 10.9 & -- & 23.8 & 39.9 \\
\hline Agriculture & 48.1 & 6.1 & 14.3 & 12.4 & -- & 30.4 & 49.2 \\
\hline Transportation and communication & -14.1 & 17.0 & 12.9 & 10.6 & -- & 20.4 & 28.2 \\
\hline Construction & -3.0 & -2.8 & 4.9 & 25.5 & -- & 40.8 & 33.7 \\
\hline Trade & 55.6 & 1.1 & 25.5 & 17.0 & -- & 176.8 & 29.5 \\
\hline Hotel industry & 183.9 & -8.3 & 33.2 & 1.8 & -- & 19.1 & 31.5 \\
\hline Health and social services & 1.5 & 3.2 & 9.9 & 11.6 & -- & 21.8 & 39.0 \\
\hline Education & 7.1 & 10.3 & 5.9 & 2.3 & -- & 25.1 & 36.1 \\
\hline Banking and insurance & 77.6 & 7.1 & 14.4 & 40.9 & -- & -3.9 & 11.4 \\
\hline Government administration & 10.1 & 7.0 & 14.9 & 16.5 & -- & 21.0 & 25.8 \\
\hline \multicolumn{8}{|l|}{ Memorandum items: } \\
\hline Average annual wage (U.S. dollars) & 44.7 & 49.5 & 55.8 & 64.8 & 76.1 & 78.8 & 90.6 \\
\hline Agriculture/Banking and insurance ratio & 0.11 & 0.11 & 0.11 & 0.09 & 0.09 & 0.12 & 0.12 \\
\hline
\end{tabular}


Table 7. Azerbaijan: Labor Market Indicators, 1999-2004

\begin{tabular}{|c|c|c|c|c|c|c|c|}
\hline & \multirow{2}{*}{1999} & \multirow[t]{2}{*}{2000} & \multirow[t]{2}{*}{2001} & \multirow[t]{2}{*}{2002} & \multicolumn{2}{|l|}{2003} & \multirow{2}{*}{$\frac{2004}{\text { First half }}$} \\
\hline & & & & & First half & Year & \\
\hline & \multicolumn{7}{|c|}{ (In thousands of persons) } \\
\hline Population & 8,016 & 8,081 & 8,141 & 8,203 & 8,229 & 8,266 & 8,300 \\
\hline Working age population & 4,615 & 4,730 & 4,858 & 4,895 & 4,911 & 5,238 & 5,260 \\
\hline Labor force & 3,748 & 3,748 & 3,763 & 3,778 & 3,788 & 3,801 & 3,805 \\
\hline Total employment & 3,707 & 3,705 & 3,715 & 3,727 & 3,735 & 3,747 & 3,750 \\
\hline Industry & 259 & 250 & 247 & 252 & 252 & 252 & 251 \\
\hline Government & 728 & 745 & 682 & 759 & 768 & 769 & 767 \\
\hline Agriculture & 1,566 & 1,517 & 1,482 & 1,495 & 1,496 & 1,497 & 1,498 \\
\hline Other & 1,154 & 1,193 & 1,304 & 1,221 & 1,219 & 1,229 & 1,234 \\
\hline Registered unemployed & 41 & 43 & 48 & 51 & 53 & 54 & 55 \\
\hline Unemployment rate (in percent of labor force) & 1.1 & 1.1 & 1.3 & 1.3 & 1.4 & 1.4 & 1.4 \\
\hline \multirow[t]{2}{*}{ Pensioners } & 1,202 & 1,219 & 1,245 & 1,276 & 1,280 & 1,280 & 1,287 \\
\hline & \multicolumn{7}{|c|}{ (Percentage changes) } \\
\hline Population & 0.8 & 0.8 & 0.7 & 0.8 & -- & 0.8 & 0.9 \\
\hline Working age population & 2.3 & 2.5 & 2.7 & 0.8 & - & 7.0 & 7.1 \\
\hline Labor force & 0.1 & 0.0 & 0.4 & 0.4 & - & 0.6 & 0.4 \\
\hline Total employment & 0.1 & -0.1 & 0.3 & 0.3 & -- & 0.5 & 0.4 \\
\hline
\end{tabular}


Table 8. Azerbaijan: Consumer Price Index, 2000-04

\begin{tabular}{|c|c|c|c|c|c|c|}
\hline & \multirow[b]{3}{*}{$\begin{array}{r}\text { Index } \\
\text { Dec. } 1994=100\end{array}$} & \multicolumn{5}{|c|}{ Percentage changes } \\
\hline & & \multicolumn{3}{|c|}{ Quarterly } & \multicolumn{2}{|c|}{ Annual } \\
\hline & & $\begin{array}{l}\text { In the } \\
\text { month }\end{array}$ & $\begin{array}{l}\text { End of } \\
\text { Period }\end{array}$ & Average & $\begin{array}{l}\text { End of } \\
\text { Period }\end{array}$ & Average \\
\hline 2000 January & 183.3 & 0.7 & & & 0.7 & -7.8 \\
\hline February & 185.2 & 1.0 & & & 2.2 & -6.9 \\
\hline March & 185.3 & 0.1 & 1.8 & 1.8 & 2.2 & -5.9 \\
\hline April & 185.3 & 0.0 & & & 2.0 & -4.8 \\
\hline May & 184.4 & -0.5 & & & 1.7 & -3.8 \\
\hline June & 182.9 & -0.8 & -1.3 & -0.2 & 1.7 & -2.9 \\
\hline July & 181.9 & -0.5 & & & 2.0 & -1.9 \\
\hline August & 181.6 & -0.2 & & & 1.8 & -0.9 \\
\hline September & 182.9 & 0.7 & 0.0 & -1.1 & 1.4 & 0.0 \\
\hline October & 183.9 & 0.6 & & & 1.7 & 0.8 \\
\hline November & 184.8 & 0.4 & & & 2.0 & 1.6 \\
\hline December & 186.0 & 0.6 & 1.7 & 1.5 & 2.2 & 1.8 \\
\hline 2001 January & 186.6 & 0.3 & & & 1.8 & 1.9 \\
\hline February & 187.6 & 0.6 & & & 1.3 & 1.8 \\
\hline March & 188.2 & 0.3 & 1.2 & 1.4 & 1.6 & 1.8 \\
\hline April & 188.5 & 0.2 & & & 1.7 & 1.7 \\
\hline May & 187.5 & -0.5 & & & 1.7 & 1.7 \\
\hline June & 186.8 & -0.4 & -0.7 & 0.1 & 2.2 & 1.8 \\
\hline July & 185.3 & -0.8 & & & 1.8 & 1.8 \\
\hline August & 184.3 & -0.5 & & & 1.5 & 1.7 \\
\hline September & 185.2 & 0.5 & -0.9 & -1.4 & 1.2 & 1.7 \\
\hline October & 186.2 & 0.6 & & & 1.2 & 1.7 \\
\hline November & 186.8 & 0.3 & & & 1.1 & 1.6 \\
\hline December & 188.5 & 0.9 & 1.8 & 1.2 & 1.3 & 1.5 \\
\hline 2002 January & 189.7 & 0.6 & & & 1.7 & 1.5 \\
\hline February & 190.5 & 0.4 & & & 1.5 & 1.6 \\
\hline March & 191.3 & 0.4 & 1.5 & 1.8 & 1.6 & 1.6 \\
\hline April & 193.1 & 0.9 & & & 2.4 & 1.6 \\
\hline May & 194.0 & 0.5 & & & 3.5 & 1.8 \\
\hline June & 192.0 & -1.0 & 0.4 & 1.3 & 2.8 & 1.8 \\
\hline July & 191.2 & -0.4 & & & 3.2 & 1.9 \\
\hline August & 190.2 & -0.6 & & & 3.2 & 2.1 \\
\hline September & 191.0 & 0.5 & -0.5 & -1.2 & 3.2 & 2.2 \\
\hline October & 192.2 & 0.6 & & & 3.2 & 2.4 \\
\hline November & 193.6 & 0.7 & & & 3.6 & 2.6 \\
\hline December & 194.8 & 0.6 & 2.0 & 1.4 & 3.3 & 2.8 \\
\hline 2003 January & 195.4 & 0.3 & & & 3.0 & 2.9 \\
\hline February & 195.6 & 0.1 & & & 2.7 & 3.0 \\
\hline March & 195.7 & 0.0 & 0.5 & 1.1 & 2.3 & 3.0 \\
\hline April & 195.9 & 0.1 & & & 1.5 & 3.0 \\
\hline May & 196.7 & 0.4 & & & 1.4 & 2.8 \\
\hline June & 195.7 & -0.5 & 0.0 & 0.3 & 1.9 & 2.7 \\
\hline July & 193.8 & -1.0 & & & 1.4 & 2.5 \\
\hline August & 193.3 & -0.3 & & & 1.6 & 2.4 \\
\hline September & 195.4 & 1.1 & -0.2 & -1.0 & 2.3 & 2.3 \\
\hline October & 197.2 & 1.0 & & & 2.6 & 2.3 \\
\hline November & 198.7 & 0.7 & & & 2.6 & 2.2 \\
\hline December & 201.7 & 1.5 & 3.2 & 2.6 & 3.6 & 2.2 \\
\hline 2004 January & 205.7 & 2.0 & & & 5.3 & 2.4 \\
\hline February & 206.0 & 0.1 & & & 5.3 & 2.7 \\
\hline March & 208.0 & 1.0 & 3.1 & 3.7 & 6.3 & 3.0 \\
\hline April & 209.3 & 0.7 & & & 6.8 & 3.4 \\
\hline May & 209.6 & 0.1 & & & 6.6 & 3.9 \\
\hline June & 206.4 & -1.5 & -0.7 & 0.9 & 5.5 & 4.2 \\
\hline July & 204.0 & -1.2 & & & 5.3 & 4.5 \\
\hline August & 203.5 & -0.2 & & & 5.3 & 4.8 \\
\hline September & 204.9 & 0.7 & -0.7 & -2.1 & 4.9 & 5.0 \\
\hline October & 213.9 & 4.4 & & & 8.5 & 5.5 \\
\hline
\end{tabular}

Source: Azerbaijan State Statistics Committee. 
Table 9. Azerbaijan: Breakdown of Consumer Price Index, 2000-04

\begin{tabular}{|c|c|c|c|c|c|c|c|c|c|c|}
\hline & \multicolumn{2}{|c|}{ Total } & \multicolumn{2}{|c|}{ Food items } & \multicolumn{2}{|c|}{ Non-food items } & \multicolumn{2}{|c|}{ Non-food goods } & \multicolumn{2}{|c|}{ Services } \\
\hline & $\begin{array}{r}\text { Percentage } \\
\text { change } \\
\end{array}$ & $\begin{array}{r}\text { Index } \\
\text { Dec. } 94=100 \\
\end{array}$ & $\begin{array}{r}\text { Percentage } \\
\text { change } \\
\end{array}$ & $\begin{array}{r}\text { Index } \\
\text { Dec. } 94=100 \\
\end{array}$ & $\begin{array}{r}\text { Percentage } \\
\text { change } \\
\end{array}$ & $\begin{array}{r}\text { Index } \\
\text { Dec. } 94=100 \\
\end{array}$ & $\begin{array}{r}\text { Percentage } \\
\text { change }\end{array}$ & $\begin{array}{r}\text { Index } \\
\text { Dec. } 94=100 \\
\end{array}$ & $\begin{array}{r}\text { Percentage } \\
\text { change }\end{array}$ & $\begin{array}{r}\text { Index } \\
\text { Dec. } 94=100 \\
\end{array}$ \\
\hline 2000 January & 0.7 & 183.3 & 0.4 & 169.7 & 1.2 & 236.6 & 2.0 & 182.9 & 0.2 & 416.7 \\
\hline February & 1.0 & 185.2 & 1.7 & 172.7 & -0.2 & 236.3 & 0.1 & 183.0 & -0.5 & 414.6 \\
\hline March & 0.1 & 185.3 & 1.1 & 174.7 & -1.8 & 232.0 & -0.5 & 182.1 & -3.6 & 399.7 \\
\hline April & 0.0 & 185.3 & 0.2 & 175.0 & -0.3 & 231.4 & -0.2 & 181.7 & -0.4 & 397.9 \\
\hline May & -0.5 & 184.4 & -0.7 & 173.8 & -0.1 & 231.1 & -0.1 & 181.4 & -0.1 & 397.6 \\
\hline June & -0.8 & 182.9 & -1.3 & 171.5 & 0.0 & 231.0 & 0.0 & 181.3 & 0.0 & 397.6 \\
\hline July & -0.5 & 181.9 & -0.8 & 170.2 & 0.0 & 230.9 & -0.1 & 181.2 & 0.0 & 397.5 \\
\hline August & -0.2 & 181.6 & -0.3 & 169.7 & 0.0 & 230.9 & 0.0 & 181.2 & 0.0 & 397.5 \\
\hline September & 0.7 & 182.9 & 1.1 & 171.6 & 0.1 & 231.1 & 0.1 & 181.5 & 0.0 & 397.5 \\
\hline October & 0.6 & 183.9 & 0.8 & 172.9 & 0.3 & 231.7 & 0.4 & 182.3 & 0.0 & 397.5 \\
\hline November & 0.4 & 184.8 & 0.7 & 174.0 & 0.0 & 231.8 & 0.1 & 182.4 & 0.0 & 397.5 \\
\hline December & 0.6 & 186.0 & 1.0 & 175.8 & 0.0 & 231.8 & 0.0 & 182.4 & 0.0 & 397.5 \\
\hline 2001 January & 0.3 & 186.6 & 0.6 & 176.9 & -0.2 & 231.2 & -0.4 & 181.6 & 0.0 & 397.6 \\
\hline February & 0.6 & 187.6 & 1.0 & 178.7 & -0.1 & 231.0 & 0.0 & 181.7 & -0.3 & 396.5 \\
\hline March & 0.3 & 188.2 & 0.5 & 179.5 & 0.0 & 230.9 & 0.0 & 181.7 & -0.1 & 396.2 \\
\hline April & 0.2 & 188.5 & 0.4 & 180.3 & -0.2 & 230.4 & -0.4 & 181.0 & 0.0 & 396.3 \\
\hline May & -0.5 & 187.5 & -0.9 & 178.7 & 0.1 & 230.6 & 0.2 & 181.3 & 0.0 & 396.3 \\
\hline June & -0.4 & 186.8 & -0.5 & 177.7 & -0.1 & 230.4 & 0.0 & 181.3 & -0.2 & 395.3 \\
\hline July & -0.8 & 185.3 & -1.4 & 175.2 & 0.1 & 230.7 & 0.2 & 181.6 & 0.0 & 395.3 \\
\hline August & -0.5 & 184.3 & -0.7 & 174.0 & -0.2 & 230.1 & -0.4 & 180.9 & 0.0 & 395.3 \\
\hline September & 0.5 & 185.2 & 0.5 & 174.8 & 0.4 & 231.1 & 0.7 & 182.3 & 0.0 & 395.3 \\
\hline October & 0.6 & 186.2 & 0.5 & 175.7 & 0.7 & 232.6 & 1.1 & 184.3 & 0.0 & 395.2 \\
\hline November & 0.3 & 186.8 & 0.4 & 176.5 & 0.1 & 232.9 & 0.2 & 184.7 & 0.0 & 395.2 \\
\hline December & 0.9 & 188.5 & 1.4 & 179.0 & 0.0 & 233.0 & 0.0 & 184.8 & 0.0 & 395.2 \\
\hline 2002 January & 0.6 & 189.7 & 0.8 & 180.5 & 0.3 & 233.7 & 0.5 & 185.8 & 0.0 & 395.3 \\
\hline February & 0.4 & 190.5 & 0.7 & 181.7 & 0.0 & 233.8 & 0.0 & 185.8 & 0.0 & 395.3 \\
\hline March & 0.4 & 191.3 & 0.6 & 182.9 & 0.0 & 233.8 & 0.0 & 185.9 & -0.1 & 394.9 \\
\hline April & 0.9 & 193.1 & 1.4 & 185.5 & 0.1 & 234.1 & 0.0 & 186.0 & 0.3 & 395.9 \\
\hline May & 0.5 & 194.0 & 0.7 & 186.9 & 0.1 & 234.2 & 0.1 & 186.2 & -0.1 & 395.7 \\
\hline June & -1.0 & 192.0 & -1.5 & 184.0 & -0.1 & 233.9 & -0.1 & 185.9 & -0.1 & 395.4 \\
\hline July & -0.4 & 191.2 & -0.6 & 182.9 & -0.1 & 233.8 & -0.1 & 185.8 & 0.0 & 395.3 \\
\hline August & -0.6 & 190.2 & -0.8 & 181.3 & -0.1 & 233.6 & 0.0 & 185.7 & -0.1 & 394.9 \\
\hline September & 0.5 & 191.0 & 0.5 & 182.3 & 0.3 & 234.3 & 0.5 & 186.6 & 0.0 & 394.9 \\
\hline October & 0.6 & 192.2 & 0.9 & 183.9 & 0.1 & 234.6 & 0.2 & 187.0 & 0.0 & 394.9 \\
\hline November & 0.7 & 193.6 & 1.0 & 185.8 & 0.3 & 235.3 & 0.5 & 188.0 & 0.0 & 394.8 \\
\hline December & 0.6 & 194.8 & 0.9 & 187.5 & 0.0 & 235.3 & 0.0 & 188.0 & 0.0 & 394.8 \\
\hline 2003 January & 0.3 & 195.4 & 0.5 & 188.4 & 0.0 & 235.4 & 0.0 & 188.0 & 0.1 & 395.0 \\
\hline February & 0.1 & 195.6 & 0.2 & 188.9 & 0.0 & 235.2 & 0.0 & 187.9 & -0.1 & 394.7 \\
\hline March & 0.0 & 195.7 & 0.1 & 189.1 & -0.1 & 235.0 & 0.0 & 187.9 & -0.2 & 394.0 \\
\hline April & 0.1 & 195.9 & 0.2 & 189.4 & 0.0 & 235.1 & 0.0 & 187.9 & 0.0 & 394.0 \\
\hline May & 0.4 & 196.7 & 0.6 & 190.5 & 0.1 & 235.3 & 0.1 & 188.2 & 0.0 & 394.1 \\
\hline June & -0.5 & 195.7 & -0.7 & 189.1 & -0.1 & 235.0 & -0.2 & 187.8 & 0.0 & 394.0 \\
\hline July & -1.0 & 193.8 & -1.6 & 186.1 & 0.1 & 235.2 & 0.1 & 188.1 & 0.0 & 394.0 \\
\hline August & -0.3 & 193.3 & -0.4 & 185.4 & -0.1 & 235.1 & -0.1 & 187.9 & 0.0 & 394.0 \\
\hline September & 1.1 & 195.4 & 1.7 & 188.5 & 0.0 & 235.1 & 0.0 & 187.9 & 0.0 & 394.0 \\
\hline October & 1.0 & 197.2 & 1.4 & 191.2 & 0.2 & 235.6 & 0.4 & 188.6 & 0.0 & 394.0 \\
\hline November & 0.7 & 198.7 & 1.0 & 193.0 & 0.3 & 236.3 & 0.6 & 189.7 & 0.0 & 394.0 \\
\hline December & 1.5 & 201.7 & 2.4 & 197.6 & 0.0 & 236.4 & 0.1 & 189.9 & 0.0 & 393.8 \\
\hline 2004 January & 2.0 & 205.7 & 3.2 & 203.9 & -0.1 & 236.3 & -0.1 & 189.6 & 0.0 & 393.8 \\
\hline February & 0.1 & 206.0 & 0.2 & 204.3 & 0.0 & 236.2 & -0.1 & 189.4 & 0.1 & 394.0 \\
\hline March & 1.0 & 208.0 & 1.6 & 207.6 & -0.1 & 235.9 & -0.2 & 189.1 & 0.0 & 394.0 \\
\hline April & 0.7 & 209.3 & 1.0 & 209.6 & 0.1 & 236.2 & 0.2 & 189.5 & -0.1 & 393.7 \\
\hline May & 0.1 & 209.6 & 0.2 & 210.0 & 0.1 & 236.3 & 0.1 & 189.7 & 0.0 & 393.7 \\
\hline June & -1.5 & 206.4 & -2.3 & 205.1 & -0.1 & 236.1 & -0.1 & 189.5 & 0.0 & 393.7 \\
\hline July & -1.2 & 204.0 & -1.8 & 201.3 & 0.0 & 236.1 & 0.0 & 189.5 & 0.0 & 393.7 \\
\hline August & -0.2 & 203.5 & -0.4 & 200.5 & 0.0 & 236.2 & 0.0 & 189.5 & 0.0 & 393.7 \\
\hline September & 0.7 & 204.9 & 1.1 & 202.7 & 0.0 & 236.1 & 0.1 & 189.6 & -0.2 & 393.1 \\
\hline October & 4.4 & 213.9 & 6.5 & 216.0 & 0.7 & 237.7 & 1.1 & 191.8 & 0.0 & 393.1 \\
\hline
\end{tabular}

Source: Azerbaijan State Statistics Committee. 
Table 10. Azerbaijan: Prices of Electricity, 1999-2003 (In manat per kilowatt-hour)

\begin{tabular}{lccccc}
\hline & 1999 & 2000 & 2001 & 2002 & 2003 \\
\hline Population & 96 & 96 & 96 & 96 & 96 \\
Industry and construction & 192 & 174 & 130 & 130 & 130 \\
Budgetary organizations & 192 & 174 & 130 & 130 & 130 \\
Apsheron Regional Water Company & 158 & 157 & 130 & 130 & 130 \\
Agriculture & 168 & 162 & 130 & 130 & 130 \\
Electrified transport & 220 & 178 & 130 & 130 & 130 \\
Non-industrial consumption & 318 & 238 & 130 & 130 & 130 \\
Trade and services & 408 & 282 & 250 & 250 & 250 \\
\hline
\end{tabular}

Source: Ministry of Economic Development. 
Table 11. Azerbaijan: Prices of Gas, 1999-2004 (In manat per thousand cubic meters)

\begin{tabular}{lrrrrrr}
\hline & 1999 & 2000 & 2001 & 2002 & 2003 & $20041 /$ \\
\hline Population & 35,560 & 35,560 & 35,560 & 35,560 & 35,560 & 81,000 \\
Communal & 108,103 & 108,103 & 106,301 & 106,301 & 106,301 & 236,000 \\
Industry & 240,000 & 240,000 & 236,000 & 236,000 & 236,000 & 236,000 \\
Azerenergy & 198,000 & 198,000 & 194,700 & 194,700 & 194,700 & 236,000 \\
\hline
\end{tabular}

Source: Ministry of Economic Development.

1/ As of November 2, 2004 
Table 12. Azerbaijan: Consolidated Government Operations, 1999-2004

(In billions of manats)

\begin{tabular}{|c|c|c|c|c|c|c|c|}
\hline & \multirow[t]{2}{*}{1999} & \multirow[t]{2}{*}{2000} & \multirow[t]{2}{*}{2001} & \multirow[t]{2}{*}{2002} & \multicolumn{2}{|c|}{2003} & \multirow{2}{*}{$\begin{array}{c}2004 \\
\text { First half }\end{array}$} \\
\hline & & & & & First half & Year & \\
\hline Total revenue and grants & 3,487 & 5,006 & 4,961 & 8,276 & 4,481 & 9,506 & 5,290 \\
\hline Total revenue & 3,425 & 4,895 & 4,934 & 8,220 & 4,481 & 9,477 & 5,290 \\
\hline Tax revenue & 2,688 & 3,414 & 3,891 & 4,575 & 2,507 & 5,494 & 2,961 \\
\hline Income tax & 816 & 1,105 & 1,062 & 1,287 & 735 & 1,644 & 1,003 \\
\hline Individual income tax & 448 & 476 & 474 & 550 & 320 & 752 & 434 \\
\hline Enterprise profits tax & 368 & 629 & 588 & 737 & 415 & 891 & 569 \\
\hline Social security contributions & 458 & 563 & 565 & 641 & 337 & 745 & 402 \\
\hline Value added tax (VAT) & 791 & 957 & 1,266 & 1,674 & 925 & 2,049 & 1,088 \\
\hline Excise taxes & 114 & 112 & 555 & 433 & 184 & 335 & 124 \\
\hline Taxes on international trade & 318 & 494 & 300 & 376 & 208 & 463 & 197 \\
\hline Other taxes & 192 & 183 & 143 & 164 & 117 & 258 & 146 \\
\hline Nontax revenue & 738 & 1,481 & 1,043 & 2,041 & 1,023 & 2,088 & 1,083 \\
\hline Of which: Oil Fund revenues & 0 & 669 & 266 & 1,153 & 583 & 1,211 & 473 \\
\hline extra-budgetary & 230 & 202 & 207 & 310 & 132 & 297 & 251 \\
\hline Tax credits for SOCAR's quasifiscal activities & $\ldots$ & $\ldots$ & $\ldots$ & 1,603 & 951 & 1,895 & 1,246 \\
\hline Total grants (current) & 61 & 111 & 28 & 57 & 0 & 28 & 0 \\
\hline Total expenditure & 4,447 & 4,914 & 4,960 & 8,388 & 4,838 & 10,114 & 4,986 \\
\hline Current expenditure & 3,754 & 4,201 & 4,412 & 6,689 & 3,490 & 7,972 & 4,365 \\
\hline Primary current expenditure & 3,679 & 4,108 & 4,343 & 5,002 & 2,496 & 5,995 & 3,081 \\
\hline Wages and salaries & 956 & 1,090 & 1,187 & 1,278 & 648 & 1,579 & 914 \\
\hline Goods and services & 1,019 & 1,139 & 1,196 & 1,353 & 766 & 2,012 & 898 \\
\hline Transfers to households & 1,349 & 1,575 & 1,665 & 1,941 & 958 & 2,192 & 1,156 \\
\hline Of which: social protection & 1,139 & 1,330 & 1,334 & 1,575 & 801 & 1,855 & 928 \\
\hline pensions & 75 & 81 & 102 & 120 & 64 & 141 & 78 \\
\hline scholarship & 19 & 48 & 14 & 16 & 8 & 17 & 10 \\
\hline other & 116 & 115 & 215 & 229 & 85 & 178 & 140 \\
\hline Subsidies & $\ldots$ & 55 & 76 & 73 & 99 & 162 & 79 \\
\hline Oil Fund operating expenditures & $\ldots$ & $\ldots$ & 0 & 3 & 2 & 4 & 1 \\
\hline Other & 336 & 249 & 220 & 354 & 23 & 46 & 32 \\
\hline Of which : extra budgetary & 252 & 202 & 195 & 303 & 106 & 0 & 125 \\
\hline SOCAR energy related subsidies & $\ldots$ & $\ldots$ & $\ldots$ & 1,603 & 951 & 1,895 & 1,246 \\
\hline Interest & 75 & 93 & 69 & 85 & 43 & 82 & 38 \\
\hline domestic & 16 & 21 & 32 & 28 & 13 & 21 & 4 \\
\hline external & 60 & 72 & 37 & 56 & 31 & 60 & 33 \\
\hline Current balance (deficit=-) & -267 & 805 & 549 & 1,587 & 991 & 1,534 & 925 \\
\hline Investment expenditure and net lending & 693 & 713 & 547 & 1,699 & 1,348 & 2,142 & 621 \\
\hline Domestically-financed & 237 & 314 & 284 & 885 & 940 & 1,522 & 477 \\
\hline Of which: Oil Fund & $\ldots$ & $\ldots$ & 4 & 189 & 202 & 600 & 309 \\
\hline Foreign-financed & 456 & 399 & 264 & 814 & 409 & 620 & 144 \\
\hline Statistical discrepancy & -65 & 229 & 116 & 32 & -85 & -184 & -146 \\
\hline Consolidated government deficit, cash basis & -895 & -137 & -115 & -144 & -273 & -425 & 450 \\
\hline Excluding Oil Fund (general government) & -895 & -806 & -376 & $-1,105$ & -652 & $-1,032$ & 287 \\
\hline Excluding foreign project loans & -439 & 262 & 149 & 669 & 135 & 195 & 595 \\
\hline Excluding grants & -956 & -248 & -143 & -201 & -273 & -453 & 304 \\
\hline Financing & 895 & 137 & 115 & 142 & 273 & 425 & -450 \\
\hline Domestic (net) & -434 & -686 & -161 & -793 & -536 & -611 & -595 \\
\hline Banking system & -308 & 438 & 62 & 90 & -137 & -73 & -548 \\
\hline Of which: Central bank & -345 & 228 & -104 & 23 & -68 & -69 & -105 \\
\hline Commercial banks & 37 & 210 & 167 & 67 & -69 & -4 & -442 \\
\hline Of which: Treasury bills & 36 & 41 & 54 & -50 & -2 & -34 & -80 \\
\hline Oil Fund & $\ldots$ & $-1,134$ & -344 & $-1,039$ & -473 & -640 & -116 \\
\hline Nonbank sector & 0 & 0 & -67 & 3 & 2 & 5 & 3 \\
\hline Privatizations and other sale of assets & 117 & 40 & 86 & 106 & 54 & 98 & 80 \\
\hline Other & -140 & -30 & 102 & 47 & 18 & 0 & -15 \\
\hline External (net) & 1,329 & 823 & 275 & 935 & 810 & 1,035 & 145 \\
\hline Loans & 1,340 & 850 & 287 & 993 & 837 & 1,123 & 182 \\
\hline Project loans & 457 & 394 & 282 & 848 & 429 & 666 & 175 \\
\hline Oil bonuses & 715 & 457 & 5 & 0 & 239 & 288 & 8 \\
\hline World Bank SAC & 168 & 0 & 0 & 144 & 169 & 169 & 0 \\
\hline Amortization due & -12 & -27 & -12 & -57 & -28 & -88 & -37 \\
\hline
\end{tabular}

Sources: Ministry of Finance, State Oil Fund, Social Protection Fund; and Fund staff estimates. 
Table 13. Azerbaijan: Functional Classification of State Budget Expenditure, 1999-2004

\begin{tabular}{|c|c|c|c|c|c|c|c|}
\hline & \multirow[t]{2}{*}{1999} & \multirow[t]{2}{*}{2000} & \multirow[t]{2}{*}{2001} & \multirow[t]{2}{*}{2002} & \multicolumn{2}{|c|}{2003} & \multirow{2}{*}{$\begin{array}{l}2004 \\
\text { First half }\end{array}$} \\
\hline & & & & & First half & Year & \\
\hline & \multicolumn{7}{|c|}{ (In billions of manats) } \\
\hline General government services 1 / & 256 & 262 & 304 & 358 & 196 & 489 & 231 \\
\hline Defense & 436 & 485 & 532 & 605 & 297 & 679 & 327 \\
\hline Public order and justice & 343 & 372 & 427 & 472 & 246 & 603 & 309 \\
\hline Education & 790 & 906 & 928 & 952 & 461 & 1,170 & 705 \\
\hline Health & 186 & 204 & 210 & 224 & 100 & 277 & 134 \\
\hline Social security & 606 & 697 & 731 & 951 & 455 & 1,070 & 572 \\
\hline Housing and community affairs & 71 & 94 & 84 & 100 & 57 & 147 & 62 \\
\hline Recreation and culture & 92 & 106 & 113 & 120 & 66 & 160 & 77 \\
\hline Agriculture & 155 & 170 & 171 & 227 & 99 & 268 & 124 \\
\hline Public works, transport, and communications & 104 & 180 & 256 & 343 & 104 & 220 & 136 \\
\hline Other economic services and expenditures & 149 & 325 & 275 & 305 & 394 & 795 & 363 \\
\hline Total expenditure (including investment) & 3,189 & 3,801 & 4,031 & 4,658 & 2,475 & 5,877 & 3,039 \\
\hline
\end{tabular}

Sources: Ministry of Finance; and Fund staff estimates.

1/ Presidency, Cabinet of Ministers, and Parliament. 
Table 14. Azerbaijan: Selected Fiscal Indicators, 1999-2004 (In percent of GDP)

\begin{tabular}{|c|c|c|c|c|c|c|c|}
\hline & \multirow[t]{2}{*}{1999} & \multirow[t]{2}{*}{2000} & \multirow[t]{2}{*}{2001} & \multirow[t]{2}{*}{$20021 /$} & \multicolumn{2}{|l|}{2003} & \multirow{2}{*}{$\frac{2004}{\text { First half }}$} \\
\hline & & & & & First half $2 /$ & Year & \\
\hline Total revenue and grants & 18.5 & 21.2 & 18.7 & 27.3 & 27.7 & 27.1 & 28.6 \\
\hline Total revenue & 18.1 & 20.7 & 18.6 & 27.1 & 27.7 & 27.0 & 28.6 \\
\hline Of which: tax revenue & 14.2 & 14.5 & 14.6 & 15.1 & 15.5 & 15.7 & 16.0 \\
\hline nontax revenue & 3.9 & 6.3 & 3.9 & 6.7 & 6.3 & 6.0 & 5.8 \\
\hline tax credits for SOCAR energy subsidies & $\ldots$ & $\ldots$ & $\ldots$ & 5.3 & 5.9 & 5.4 & 6.7 \\
\hline Of which: oil revenue 1/ & 4.2 & 7.5 & 6.7 & 15.1 & 16.5 & 15.2 & 14.5 \\
\hline non-oil revenue & 14.0 & 13.2 & 11.9 & 12.0 & 11.2 & 11.8 & 14.0 \\
\hline Non-oil revenue (percent of non-oil GDP) & 17.5 & 18.8 & 17.5 & 17.4 & 16.9 & 17.2 & 20.8 \\
\hline Total grants (current) & 0.3 & 0.5 & 0.1 & 0.2 & 0.0 & 0.1 & 0.0 \\
\hline Total expenditure & 23.6 & 20.8 & 18.7 & 27.7 & 29.9 & 28.9 & 26.9 \\
\hline Primary expenditure & 23.2 & 20.4 & 18.4 & 22.1 & 23.8 & 23.2 & 20.0 \\
\hline Primary current expenditure & 19.5 & 17.4 & 16.3 & 16.5 & 15.4 & 17.1 & 16.6 \\
\hline Of which: wage bill & 5.1 & 4.6 & 4.5 & 4.2 & 4.0 & 4.5 & 4.9 \\
\hline goods and services & 5.4 & 4.8 & 4.5 & 4.5 & 4.7 & 5.7 & 4.8 \\
\hline transfers & 7.1 & 6.7 & 6.3 & 6.4 & 5.9 & 6.3 & 6.2 \\
\hline other & 1.9 & 1.3 & 1.1 & 1.4 & 0.8 & 0.6 & 0.6 \\
\hline Subsidies to Azerenergy and Azerigas & $\ldots$ & $\ldots$ & $\ldots$ & 5.3 & 5.9 & 5.4 & 6.7 \\
\hline Domestically financed investment & 1.3 & 1.3 & 1.1 & 2.9 & 5.8 & 4.3 & 2.6 \\
\hline Of which: Oil Fund & & $\ldots$ & 0.0 & 0.6 & 1.2 & 1.7 & 1.7 \\
\hline Foreign-financed investment expenditure & 2.4 & 1.7 & 1.0 & 2.7 & 2.5 & 1.8 & 0.8 \\
\hline Interest on public debt & 0.4 & 0.4 & 0.3 & 0.3 & 0.3 & 0.2 & 0.2 \\
\hline Wages/ primary expenditure (in percentage) & 26.0 & 26.5 & 27.3 & 25.5 & 26.2 & 26.3 & 29.8 \\
\hline Transfers / primary expenditure (in percentage) & 36.7 & 38.3 & 38.3 & 38.8 & 38.9 & 36.6 & 37.8 \\
\hline Wages/ non-oil revenue (in percentage) & 36.2 & 34.9 & 37.5 & 35.2 & 35.8 & 38.1 & 35.1 \\
\hline Transfers / non-oil revenue (in percentage) & 51.1 & 50.4 & 52.7 & 53.4 & 52.9 & 52.9 & 44.4 \\
\hline Expenditure in education and health & 5.2 & 4.7 & 4.3 & 4.5 & 3.5 & 4.1 & 4.5 \\
\hline Education & 4.2 & 3.8 & 3.5 & 3.6 & 2.9 & 3.3 & 3.8 \\
\hline Health & 1.0 & 0.9 & 0.8 & 0.9 & 0.6 & 0.8 & 0.7 \\
\hline Military expenditure & 2.3 & 2.1 & 1.8 & 2.0 & 0.8 & 1.9 & 0.8 \\
\hline Current expenditure & 19.9 & 17.8 & 16.6 & 22.1 & 21.6 & 22.7 & 23.6 \\
\hline Investment expenditure & 3.7 & 3.0 & 2.1 & 4.8 & 5.3 & 4.5 & 3.1 \\
\hline Current balance ( $+=$ surplus) & -1.4 & 3.4 & 2.1 & 5.2 & 6.1 & 4.4 & 5.0 \\
\hline Primary balance ( $+=$ surplus) & -4.3 & -0.2 & -0.2 & -0.2 & -1.4 & -1.0 & 2.6 \\
\hline Primary balance, excluding oil ( $+=$ surplus) $1 /$ & -8.5 & -7.7 & -6.8 & -15.3 & -17.9 & -16.2 & -11.9 \\
\hline Primary balance (excl. externally financed investment) & -1.9 & 1.5 & 0.8 & 2.5 & 1.1 & 0.8 & 3.4 \\
\hline Consolidated government deficit, cash basis & -4.7 & -0.6 & -0.4 & -0.5 & -1.7 & -1.2 & 2.4 \\
\hline Excluding Oil Fund (general government) & -4.7 & -3.4 & -1.4 & -3.6 & -4.0 & -2.9 & 1.5 \\
\hline Excluding foreign project loans & -2.3 & 1.1 & 0.6 & 2.2 & 0.8 & 0.6 & 3.2 \\
\hline Excluding grants & -5.1 & -1.1 & -0.5 & -0.7 & -1.7 & -1.3 & 1.6 \\
\hline Non-oil balance & -8.9 & -8.1 & -7.1 & -10.3 & -12.3 & -11.0 & -5.4 \\
\hline \multicolumn{8}{|l|}{ Memorandum items: } \\
\hline Total external assistance, including IMF (net) & 9.1 & 2.6 & 0.6 & 2.6 & 3.4 & 1.8 & 0.5 \\
\hline Of which: IMF (net) & 2.0 & -1.0 & -0.5 & -0.6 & -0.3 & -0.6 & -0.5 \\
\hline Total external assistance, excluding IMF & 7.1 & 3.6 & 1.1 & 3.3 & 3.7 & 2.4 & 0.9 \\
\hline Project financing & 2.4 & 1.7 & 1.1 & 2.8 & 2.7 & 1.9 & 0.9 \\
\hline Program financing & 4.7 & 1.9 & 0.0 & 0.5 & 1.0 & 0.5 & 0.0 \\
\hline
\end{tabular}

Sources: Ministry of Finance, State Oil Fund, Social Protection Fund; and Fund staff estimates.

1/ Starting from 2002 estimates include SOCAR energy-related tax credit, and subsidies to Azerenergy and Azerigas.

2/ Relative to six month GDP. 
Table 15. Azerbaijan: Social Protection Fund, 1999-2004

\begin{tabular}{|c|c|c|c|c|c|c|c|}
\hline & \multirow[t]{2}{*}{1999} & \multirow[t]{2}{*}{2000} & \multirow[t]{2}{*}{2001} & \multirow[t]{2}{*}{2002} & \multicolumn{2}{|c|}{2003} & \multirow{2}{*}{$\begin{array}{c}2004 \\
\text { First half }\end{array}$} \\
\hline & & & & & First half & Year & \\
\hline & \multicolumn{6}{|c|}{ (In billions of manat) } & \\
\hline Total revenues & 1,139 & 1,330 & 1,334 & 1,605 & 840 & 1,889 & 972 \\
\hline Total payroll tax & 670 & 806 & 805 & 931 & 512 & 1,114 & 623 \\
\hline Of which: paid by non-budgetary agencies & 422 & 522 & 527 & 597 & 371 & 766 & 415 \\
\hline Transfer from State Budget & 462 & 515 & 515 & 630 & 326 & 768 & 343 \\
\hline Others & 7 & 9 & 14 & 44 & 2 & 6 & 6 \\
\hline Total expenditures & 1,139 & 1,330 & 1,334 & 1,578 & 835 & 1,884 & 965 \\
\hline Pensions & 695 & 783 & 774 & 852 & 468 & 1,046 & 553 \\
\hline Old age pensioners & 638 & 727 & 714 & 788 & 433 & 970 & 506 \\
\hline Working pensioners & 57 & 56 & 60 & 64 & 35 & 76 & 47 \\
\hline Child allowances & 8 & 8 & 6 & 6 & 4 & 7 & 3 \\
\hline Maternity leave & 9 & 11 & 11 & 11 & 5 & 12 & 7 \\
\hline Sanatorium vouchers & 15 & 19 & 17 & 19 & 10 & 20 & 12 \\
\hline Sick leave & 19 & 28 & 32 & 35 & 21 & 44 & 30 \\
\hline Funeral allowances & 10 & 9 & 9 & 11 & 6 & 11 & 6 \\
\hline Compensation for elimination of communal services & 38 & 54 & 42 & 85 & 65 & 133 & 67 \\
\hline Other compensations and allowances & 319 & 342 & 341 & 342 & 236 & 556 & 184 \\
\hline Children under 16 & 169 & 183 & 177 & 169 & 77 & 159 & 0 \\
\hline Pensioners & 136 & 142 & 145 & 150 & 155 & 388 & 180 \\
\hline War veterans & 14 & 17 & 19 & 23 & 4 & 9 & 4 \\
\hline Others & 26 & 76 & 102 & 217 & 20 & 55 & 103 \\
\hline \multirow[t]{2}{*}{ Balance } & 0 & 0 & 0 & 27 & 5 & 5 & 7 \\
\hline & \multicolumn{6}{|c|}{ (In percent of GDP) } & \\
\hline Total revenues & 6.0 & 5.6 & 5.0 & 5.3 & 5.2 & 5.4 & 5.2 \\
\hline Total payroll tax & 3.6 & 3.4 & 3.0 & 3.1 & 3.2 & 3.2 & 3.4 \\
\hline Of which: paid by non-budgetary agencies & 2.2 & 2.2 & 2.0 & 2.0 & 2.3 & 2.2 & 2.2 \\
\hline Transfer from State Budget & 2.4 & 2.2 & 1.9 & 2.1 & 2.0 & 2.2 & 1.9 \\
\hline Others & 0.0 & 0.0 & 0.0 & 0.0 & 0.0 & 0.0 & 0.0 \\
\hline Total expenditures & 6.0 & 5.6 & 5.0 & 5.2 & 5.2 & 5.4 & 5.2 \\
\hline Pensions & 3.7 & 3.3 & 2.9 & 2.8 & 2.9 & 3.0 & 3.0 \\
\hline Old age pensioners & 3.4 & 3.1 & 2.7 & 2.6 & 2.7 & 2.8 & 2.7 \\
\hline Working pensioners & 0.3 & 0.2 & 0.2 & 0.2 & 0.2 & 0.2 & 0.3 \\
\hline Child allowances & 0.0 & 0.0 & 0.0 & 0.0 & 0.0 & 0.0 & 0.0 \\
\hline Maternity leave & 0.0 & 0.0 & 0.0 & 0.0 & 0.0 & 0.0 & 0.0 \\
\hline Sanatorium vouchers & 0.1 & 0.1 & 0.1 & 0.1 & 0.1 & 0.1 & 0.1 \\
\hline Sick leave & 0.1 & 0.1 & 0.1 & 0.1 & 0.1 & 0.1 & 0.2 \\
\hline Funeral allowances & 0.1 & 0.0 & 0.0 & 0.0 & 0.0 & 0.0 & 0.0 \\
\hline Compensation for elimination of communal services & 0.2 & 0.2 & 0.2 & 0.3 & 0.4 & 0.4 & 0.4 \\
\hline Other compensations and allowances & 1.7 & 1.4 & 1.3 & 1.1 & 1.5 & 1.6 & 1.0 \\
\hline Children under 16 & 0.9 & 0.8 & 0.7 & 0.6 & 0.5 & 0.5 & 0.0 \\
\hline Pensioners & 0.7 & 0.6 & 0.5 & 0.5 & 1.0 & 1.1 & 1.0 \\
\hline War veterans & 0.1 & 0.1 & 0.1 & 0.1 & 0.0 & 0.0 & 0.0 \\
\hline Others & 0.1 & 0.3 & 0.4 & 0.7 & 0.1 & 0.2 & 0.6 \\
\hline Balance & 0.0 & 0.0 & 0.0 & 0.1 & 0.0 & 0.0 & 0.0 \\
\hline \multicolumn{8}{|l|}{ Memorandum item: } \\
\hline Nominal GDP (billions of manat) & 18,875 & 23,591 & 26,578 & 30,312 & 16,171 & 35,054 & 18,520 \\
\hline Number of pensioners (thousands) & 1,202 & 1,219 & 1,245 & 1,276 & 1,280 & 1,280 & 1,287 \\
\hline
\end{tabular}

Sources: Social Protection Fund; and Fund staff estimates. 
Table 16. Azerbaijan: Summary Accounts of the Azerbaijan National Bank, 1999-2004 1/ (In billions of manats, end of period stocks)

\begin{tabular}{|c|c|c|c|c|c|c|c|}
\hline & \multirow[t]{2}{*}{1999} & \multirow[t]{2}{*}{2000} & \multirow[t]{2}{*}{2001} & \multirow[t]{2}{*}{2002} & \multicolumn{2}{|c|}{2003} & \multirow{2}{*}{$\frac{2004}{9 \text { months }}$} \\
\hline & & & & & 9 months & Year & \\
\hline Net foreign assets & 1,158 & 1,569 & 2,045 & 2,158 & 2,454 & 2,672 & 3,638 \\
\hline Net international reserves (convertible) & 1,161 & 1,569 & 2,046 & 2,163 & 2,458 & 2,676 & 3,642 \\
\hline Gross international reserves (convertible) & 2,945 & 3,102 & 3,462 & 3,527 & 3,692 & 3,952 & 4,675 \\
\hline Foreign liabilities (convertible) & $-1,783$ & $-1,534$ & $-1,415$ & $-1,363$ & $-1,234$ & $-1,276$ & $-1,033$ \\
\hline Other & -4 & 0 & -1 & -5 & -5 & -4 & -4 \\
\hline Net domestic assets & 213 & 199 & -248 & -108 & -147 & -141 & -220 \\
\hline Domestic credit & 433 & 612 & 211 & 220 & 204 & 303 & 152 \\
\hline Net claims on general government & -317 & -90 & 110 & 133 & -22 & 65 & -123 \\
\hline Net claims on central government & 330 & 452 & 677 & 456 & 57 & 68 & -120 \\
\hline Claims on central government & 493 & 497 & 790 & 723 & 714 & 653 & 707 \\
\hline Deposits of central government & -163 & -45 & -113 & -266 & -657 & -585 & -827 \\
\hline Pre -2000 oil bonus deposits & -646 & -541 & -566 & -323 & -3 & -3 & -3 \\
\hline World Bank counterpart funds & 0 & 0 & 0 & 0 & -76 & 0 & 0 \\
\hline Claims on banks & 746 & 701 & 101 & 86 & 225 & 237 & 299 \\
\hline Credit to the economy (excluding ANB bills) & 3 & 0 & 0 & 0 & 2 & 2 & 2 \\
\hline ANB bills & & & & & & & -26 \\
\hline Other items (net) & -220 & -413 & -459 & -328 & -352 & -445 & -372 \\
\hline Reserve money & 1,370 & 1,767 & 1,797 & 2,050 & 2,306 & 2,531 & 3,417 \\
\hline Manat reserve money & 1,302 & 1,539 & 1,684 & 1,872 & 2,101 & 2,316 & 2,905 \\
\hline Currency in circulation & 1,185 & 1,420 & 1,534 & 1,756 & 1,904 & 2,169 & 2,368 \\
\hline Bank reserves & 78 & 117 & 145 & 110 & 197 & 147 & 536 \\
\hline Reserves in foreign currencies & 68 & 228 & 113 & 178 & 206 & 215 & 513 \\
\hline Other deposits & 39 & 2 & 5 & 6 & 0 & 0 & 0 \\
\hline
\end{tabular}

Source: Azerbaijan National Bank.

1/ At end of period exchange rates. 
Table 17. Azerbaijan: Summary Accounts of the Commercial Banks, 1999-2004 1/ (In billions of manats, end of period stocks)

\begin{tabular}{|c|c|c|c|c|c|c|c|}
\hline & \multirow[t]{2}{*}{1999} & \multirow{2}{*}{2000} & \multirow{2}{*}{2001} & \multirow{2}{*}{2002} & \multicolumn{2}{|c|}{2003} & \multirow{2}{*}{2004} \\
\hline & & & & & 9 months & Year & \\
\hline Net foreign assets & 231 & 54 & 480 & 494 & 520 & 574 & 970 \\
\hline Net foreign assets (hard currency) & 234 & 52 & 471 & 493 & 514 & 569 & 969 \\
\hline Assets & 419 & 493 & 910 & 1,022 & 1,028 & 1,102 & 1,342 \\
\hline Liabilities & -185 & -440 & -440 & -529 & -514 & -532 & -373 \\
\hline Claims on BRO countries & -3 & 2 & 9 & 1 & 6 & 5 & 1 \\
\hline Net domestic assets & 645 & 1,200 & 1,478 & 1,757 & 2,230 & 2,479 & 3,665 \\
\hline Domestic credit & 2,209 & 2,319 & 2,099 & 2,471 & 2,963 & 3,217 & 3,976 \\
\hline Claims on general government (net) & -39 & 106 & 273 & 340 & 250 & 271 & -240 \\
\hline Claims on central government (net) & -31 & 149 & 273 & 340 & 251 & 271 & -240 \\
\hline Claims on central government & 103 & 276 & 325 & 366 & 288 & 334 & 59 \\
\hline Deposits of central government & -134 & -127 & -51 & -26 & -37 & -63 & -298 \\
\hline Claims on Social Protection Fund (net) & -8 & -42 & 0 & 0 & 0 & 0 & 0 \\
\hline Credit to the economy & 2,248 & 2,213 & 1,826 & 2,131 & 2,713 & 2,947 & 4,216 \\
\hline Reserves & 178 & 392 & 318 & 366 & 545 & 515 & 1,310 \\
\hline Cash in national currency & 49 & 70 & 65 & 88 & 130 & 128 & 252 \\
\hline Reserves at NBA & 129 & 321 & 254 & 278 & 414 & 388 & 1,058 \\
\hline Other items, net & $-1,742$ & $-1,511$ & -940 & $-1,080$ & $-1,278$ & $-1,254$ & $-1,622$ \\
\hline Deposits in manat and foreign currency & 875 & 1,254 & 1,958 & 2,251 & 2,750 & 3,053 & 4,634 \\
\hline Deposits in manats & 290 & 276 & 279 & 351 & 552 & 551 & 927 \\
\hline State enterprises & 165 & 124 & 124 & 157 & 241 & 228 & 394 \\
\hline Private sector deposits & 124 & 152 & 155 & 195 & 312 & 324 & 533 \\
\hline Foreign currency deposits ( residents) & 585 & 978 & 1,679 & 1,900 & 2,198 & 2,502 & 3,707 \\
\hline
\end{tabular}

Source: Azerbaijan National Bank.

1/ At end of period exchange rates. 
Table 18. Azerbaijan: Net Bank Credit to the Consolidated Government, 1999-2004 1/ (In billions of manats, end of period stocks)

\begin{tabular}{|c|c|c|c|c|c|c|c|}
\hline & \multirow[t]{2}{*}{1999} & \multirow[t]{2}{*}{2000} & \multirow[t]{2}{*}{2001} & \multirow[t]{2}{*}{2002} & \multicolumn{2}{|c|}{2003} & \multirow{2}{*}{$\frac{2004}{9 \text { months }}$} \\
\hline & & & & & 9 months & Year & \\
\hline \multicolumn{8}{|l|}{ Central Bank } \\
\hline Net claims on general government & -317 & -90 & 110 & 133 & -22 & 65 & -123 \\
\hline Net claims on central government & 330 & 452 & 677 & 456 & 57 & 68 & -120 \\
\hline Claims on central government & 493 & 497 & 790 & 723 & 714 & 653 & 707 \\
\hline Deposits of central government & -163 & -45 & -113 & -266 & -657 & -585 & -827 \\
\hline Pre-2000 oil signature bonus & -646 & -541 & -566 & -323 & -3 & -3 & -3 \\
\hline World Bank counterpart funds & 0 & 0 & 0 & 0 & -76 & 0 & 0 \\
\hline \multicolumn{8}{|l|}{ Commercial banks } \\
\hline Net claims on general government & -39 & 106 & 273 & 340 & 250 & 271 & -240 \\
\hline Net claims on central government & -31 & 149 & 273 & 340 & 251 & 271 & -240 \\
\hline Claims on central government & 103 & 276 & 325 & 366 & 288 & 334 & 59 \\
\hline Deposits of central government & -134 & -127 & -51 & -26 & -37 & -63 & -298 \\
\hline Claims on Social Protection Fund (net) & -8 & -42 & 0 & 0 & 0 & 0 & 0 \\
\hline \multicolumn{8}{|l|}{ Total banking system } \\
\hline Net claims on general government & -356 & 17 & 384 & 473 & 228 & 335 & -364 \\
\hline Net claims on central government & 299 & 601 & 950 & 796 & 308 & 338 & -360 \\
\hline Claims on social protection fund (net) & -8 & -42 & 0 & 0 & 0 & 0 & 0 \\
\hline Other & -646 & -541 & -566 & -323 & -80 & -3 & -3 \\
\hline
\end{tabular}

Source: Azerbaijan National Bank.

1/ At end of period exchange rates. 
Table 19. Azerbaijan: Monetary Survey, 1999-2004 1/

\begin{tabular}{|c|c|c|c|c|c|c|c|}
\hline & \multirow[t]{2}{*}{1999} & \multirow[t]{2}{*}{2000} & \multirow[t]{2}{*}{2001} & \multirow[t]{2}{*}{2002} & \multicolumn{2}{|c|}{2003} & \multirow{2}{*}{$\frac{2004}{9 \text { months }}$} \\
\hline & & & & & 9 months & Year & \\
\hline & \multicolumn{7}{|c|}{ (In billions of manats, end of period stocks) } \\
\hline Net foreign assets & 1,388 & 1,623 & 2,525 & 2,652 & 2,973 & 3,246 & 4,607 \\
\hline Net international reserves of the ANB (convertible) & 1,161 & 1,569 & 2,046 & 2,163 & 2,458 & 2,676 & 3,642 \\
\hline Net foreign assets of commercial banks (convertible) & 234 & 52 & 471 & 493 & 514 & 569 & 969 \\
\hline Other & -7 & 2 & 8 & -4 & 1 & 1 & -4 \\
\hline Net domestic assets & 662 & 983 & 907 & 1,273 & 1,550 & 1,848 & 2,144 \\
\hline Domestic credit & 1,896 & 2,229 & 2,210 & 2,605 & 2,943 & 3,284 & 3,829 \\
\hline Net claims on general government & -356 & 17 & 384 & 473 & 228 & 335 & -364 \\
\hline Net claims on central government & 299 & 601 & 950 & 796 & 308 & 338 & -360 \\
\hline Pre-2000 oil bonus deposits & -646 & -541 & -566 & -323 & -3 & -3 & -3 \\
\hline WB counterpart funds & 0 & 0 & 0 & 0 & -76 & 0 & 0 \\
\hline Credit to the economy & 2,252 & 2,213 & 1,826 & 2,132 & 2,715 & 2,949 & 4,219 \\
\hline Other items (net) & $-1,234$ & $-1,247$ & $-1,302$ & $-1,331$ & $-1,394$ & $-1,436$ & $-1,685$ \\
\hline Broad money & 2,050 & 2,606 & 3,433 & 3,926 & 4,523 & 5,094 & 6,751 \\
\hline Manat broad money & 1,465 & 1,628 & 1,753 & 2,026 & 2,325 & 2,592 & 3,044 \\
\hline Currency outside banks & 1,136 & 1,350 & 1,469 & 1,669 & 1,773 & 2,041 & 2,117 \\
\hline Manat deposits & 329 & 278 & 284 & 357 & 552 & 551 & 927 \\
\hline \multirow[t]{2}{*}{ Foreign currency deposits } & 585 & 978 & 1,679 & 1,900 & 2,198 & 2,502 & 3,707 \\
\hline & \multicolumn{7}{|c|}{ (Changes in percent of beginning of the period money stock, unless otherwise specified) } \\
\hline Net foreign assets & 41.9 & 11.5 & 34.6 & 3.7 & 8.2 & 15.1 & 26.7 \\
\hline Net domestic assets & -33.2 & 15.6 & -2.9 & 10.7 & 7.0 & 14.6 & 5.8 \\
\hline Domestic credit & -18.0 & 16.3 & -0.8 & 11.5 & 8.6 & 17.3 & 10.7 \\
\hline Credit to the economy & 4.8 & -1.9 & -14.8 & 8.9 & 14.9 & 20.8 & 24.9 \\
\hline Credit to the economy (annual change) & 4.2 & -1.7 & -17.5 & 16.7 & 38.6 & 38.3 & 55.4 \\
\hline Broad money (percentage change) & 8.7 & 27.1 & 31.7 & 14.4 & 15.2 & 29.8 & 32.5 \\
\hline Average broad money (percentage change) & -0.3 & 12.9 & 24.7 & 24.6 & $\ldots$ & 21.1 & $\ldots$ \\
\hline Manat broad money (annual change) & 5.3 & 11.1 & 7.7 & 15.5 & 31.6 & 28.0 & 30.9 \\
\hline Currency as a ratio to broad money & 55.4 & 51.8 & 42.8 & 42.5 & 39.2 & 40.1 & 31.4 \\
\hline Manat deposits as a ratio to broad money & 16.1 & 10.7 & 8.3 & 9.1 & 12.2 & 10.8 & 13.7 \\
\hline Foreign currency deposits as a ratio to broad money & 28.5 & 37.5 & 48.9 & 48.4 & 48.6 & 49.1 & 54.9 \\
\hline \multicolumn{8}{|l|}{ Memorandum Items: } \\
\hline Gross international reserves (US\$ millions) & 673 & 680 & 725 & 721 & 752 & 803 & 952 \\
\hline Net international reserves (US\$ millions) & 264 & 344 & 428 & 441 & 500 & 543 & 741 \\
\hline Exchange rate (Manat/U.S. dollar, end of period) & 4,378 & 4,565 & 4,775 & 4,893 & 4,910 & 4,923 & 4,908 \\
\hline Exchange rate (Manat/U.S. dollar, period average) & 4,120 & 4,474 & 4,657 & 4,861 & 4,914 & 4,911 & 4,907 \\
\hline Velocity of manat broad money $2 /$ & 13.2 & 16.2 & 16.6 & 16.8 & 15.8 & 15.8 & 16.1 \\
\hline Money multiplier & 1.5 & 1.5 & 1.9 & 1.9 & 2.0 & 2.0 & 2.0 \\
\hline
\end{tabular}

Sources: Azerbaijan National Bank.

1/ At end of period exchange rates

2 / Velocity is defined as nominal GDP divided by average manat broad money. 
Table 20. Azerbaijan: Credit and Deposit Interest Rates, 2000-04

(In percent per year)

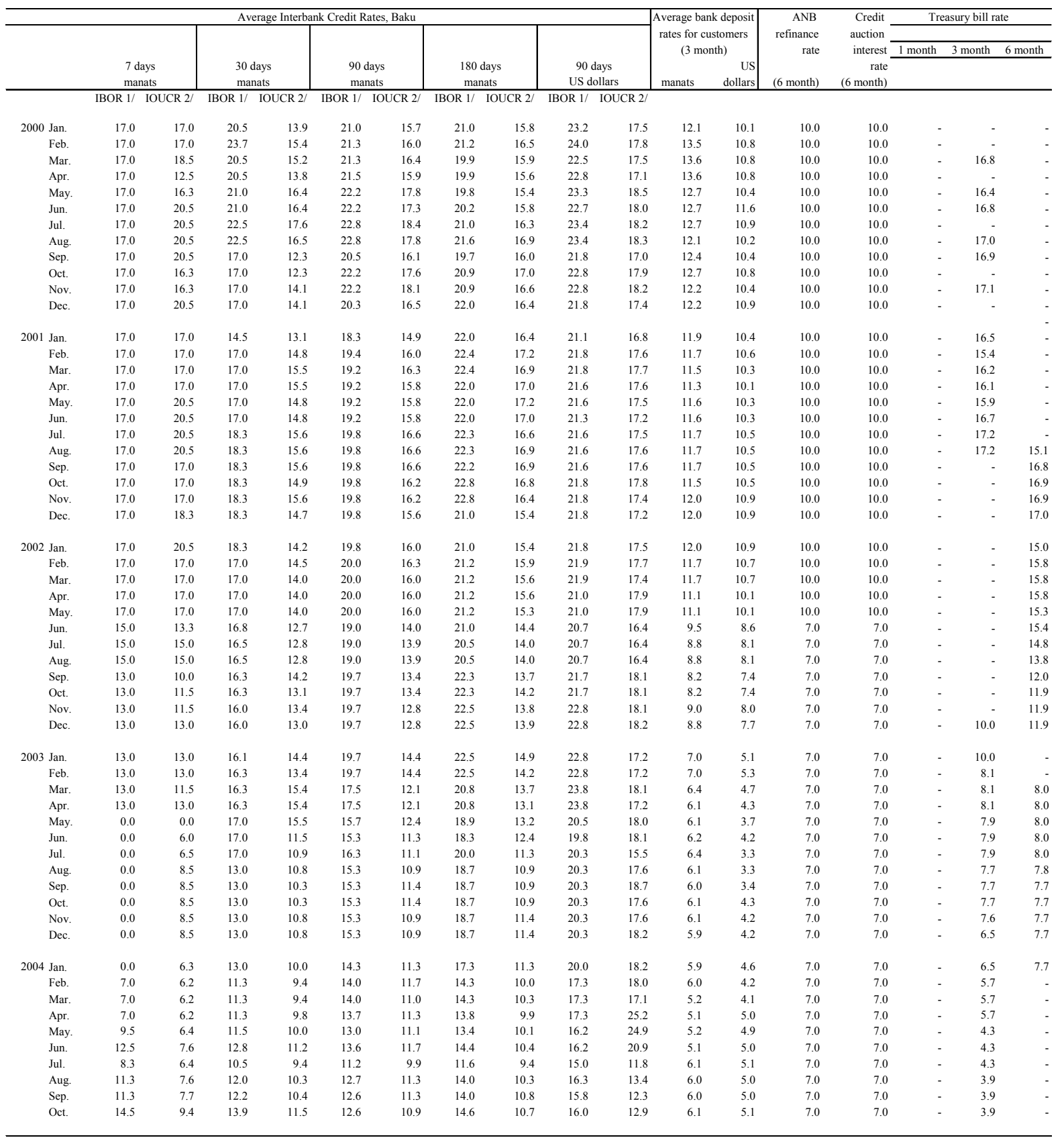

Source: Baku Interbank Currency Exchange.

1/ IBOR: quoted interbank offer rate.

2/ IOUCR: average interbank offer rate. 
Table 21. Azerbaijan: Exchange Rates, 1998-2004 (Manat per U.S. dollar)

\begin{tabular}{|c|c|c|c|c|c|}
\hline & & $\begin{array}{r}\text { Period } \\
\text { average }\end{array}$ & $\begin{array}{r}\text { Percentage } \\
\text { change }\end{array}$ & $\begin{array}{l}\text { End of } \\
\text { period }\end{array}$ & $\begin{array}{r}\text { Percentage } \\
\text { change }\end{array}$ \\
\hline \multirow[t]{5}{*}{1998} & QI & 3,884 & -0.5 & 3,868 & -0.5 \\
\hline & QII & 3,861 & -0.6 & 3,861 & -0.2 \\
\hline & QIII & 3,862 & 0.0 & 3,857 & -0.1 \\
\hline & QIV & 3,868 & 0.2 & 3,890 & 0.9 \\
\hline & Year & 3,869 & -2.9 & 3,890 & 0.1 \\
\hline \multirow[t]{5}{*}{1999} & QI & 3,910 & 1.1 & 3,929 & 1.0 \\
\hline & QII & 3,959 & 1.2 & 3,975 & 1.2 \\
\hline & QIII & 4,261 & 7.6 & 4,320 & 8.7 \\
\hline & QIV & 4,351 & 2.1 & 4,378 & 1.3 \\
\hline & Year & 4,120 & 6.5 & 4,378 & 12.5 \\
\hline \multirow[t]{5}{*}{2000} & QI & 4,395 & 1.0 & 4,413 & 0.8 \\
\hline & QII & 4,446 & 1.2 & 4,476 & 1.4 \\
\hline & QIII & 4,502 & 1.3 & 4,527 & 1.1 \\
\hline & QIV & 4,554 & 1.1 & 4,565 & 0.8 \\
\hline & Year & 4,474 & 8.6 & 4,565 & 4.3 \\
\hline \multirow[t]{5}{*}{2001} & QI & 4,586 & 0.7 & 4,606 & 0.9 \\
\hline & QII & 4,629 & 0.9 & 4,648 & 0.9 \\
\hline & QIII & 4,674 & 1.0 & 4,693 & 1.0 \\
\hline & QIV & 4,737 & 1.3 & 4,775 & 1.7 \\
\hline & Year & 4,657 & 4.1 & 4,775 & 4.6 \\
\hline \multirow[t]{5}{*}{2002} & QI & 4,807 & 1.5 & 4,825 & 1.0 \\
\hline & QII & 4,851 & 0.9 & 4,870 & 0.9 \\
\hline & QIII & 4,890 & 0.8 & 4,897 & 0.6 \\
\hline & QIV & 4,894 & 0.1 & 4,893 & -0.1 \\
\hline & Year & 4,861 & 4.4 & 4,893 & 2.5 \\
\hline \multirow[t]{5}{*}{2003} & QI & 4,900 & 0.1 & 4,907 & 0.3 \\
\hline & QII & 4,914 & 0.3 & 4,913 & 0.1 \\
\hline & QIII & 4,915 & 0.0 & 4,910 & -0.1 \\
\hline & QIV & 4,915 & 0.0 & 4,923 & 0.3 \\
\hline & Year & 4,911 & 1.0 & 4,923 & 0.6 \\
\hline \multirow[t]{3}{*}{2004} & QI & 4,932 & 0.3 & 4,925 & 0.0 \\
\hline & QII & 4,914 & -0.4 & 4,905 & -0.4 \\
\hline & QIII & 4,905 & -0.2 & 4,908 & 0.1 \\
\hline
\end{tabular}

Sources: Azerbaijan National Bank; and Fund staff calculations. 
Table 22. Azerbaijan: Balance of Payments, 1999-2004

(In millions of U.S. dollars)

\begin{tabular}{|c|c|c|c|c|c|c|c|}
\hline & \multirow[t]{2}{*}{1999} & \multirow[t]{2}{*}{2000} & \multirow[t]{2}{*}{2001} & \multirow[t]{2}{*}{2002} & \multicolumn{2}{|c|}{2003} & \multirow{2}{*}{$\begin{array}{c}2004 \\
\text { First half }\end{array}$} \\
\hline & & & & & First half & Year & \\
\hline Exports, f.o.b. & 1,025 & 1,799 & 2,046 & 2,305 & 1,254 & 2,625 & 1,679 \\
\hline Of which: oil and other hydrocarbon products & 801 & 1,519 & 1,841 & 2,046 & 1,106 & 2,250 & 1,286 \\
\hline other & 224 & 279 & 205 & 259 & 147 & 374 & 393 \\
\hline Imports, f.o.b. & $-1,433$ & $-1,539$ & $-1,465$ & $-1,823$ & $-1,164$ & $-2,723$ & $-1,553$ \\
\hline Of which: oil sector & -195 & -147 & -138 & -336 & -433 & $-1,109$ & -616 \\
\hline other & $-1,239$ & $-1,393$ & $-1,327$ & $-1,487$ & -731 & $-1,614$ & -937 \\
\hline Trade balance & -408 & 260 & 581 & 482 & 89 & -98 & 126 \\
\hline Services (net) & -228 & -225 & -375 & -936 & -757 & $-1,614$ & $-1,184$ \\
\hline Credit & 257 & 260 & 290 & 362 & 202 & 431 & 227 \\
\hline Debit & -485 & -485 & -665 & $-1,298$ & -959 & $-2,045$ & $-1,411$ \\
\hline Of which: oil sector & -189 & -154 & -329 & -868 & -734 & $-1,505$ & $-1,057$ \\
\hline Income & -45 & -295 & -332 & -386 & -194 & -442 & -314 \\
\hline Investment income (net) & 9 & -222 & -256 & -326 & -164 & -374 & -245 \\
\hline Of which: profit of oil consortium & -23 & -278 & -291 & -344 & -189 & -386 & -227 \\
\hline Compensation of employees & -32 & -47 & -47 & -39 & -21 & -50 & -49 \\
\hline Interest on public debt (including Fund) & -22 & -26 & -29 & -20 & -10 & -19 & -21 \\
\hline Transfers (net) & 82 & 73 & 77 & 70 & 62 & 134 & 106 \\
\hline Private & 25 & 11 & 13 & 15 & 39 & 77 & 71 \\
\hline Public & 56 & 62 & 64 & 55 & 23 & 57 & 36 \\
\hline Current account balance & -600 & -187 & -50 & -769 & -800 & $-2,021$ & $-1,266$ \\
\hline Net direct investment & 510 & 149 & 299 & 1,048 & 984 & 2,353 & 1,345 \\
\hline Oil companies & 349 & 14 & 197 & 984 & 970 & 2,315 & 1,317 \\
\hline contracted (net) & 527 & 454 & 732 & 1,613 & 1,271 & 2,972 & 1,857 \\
\hline capital repatriation & -333 & -541 & -542 & -629 & -350 & -716 & -542 \\
\hline bonus & 155 & 101 & 7 & 0 & 49 & 60 & 2 \\
\hline Other (net) & 161 & 135 & 101 & 64 & 15 & 38 & 28 \\
\hline Public sector capital & 215 & 245 & 140 & 167 & 100 & 115 & 7 \\
\hline Medium- and Long-Term borrowing & 228 & 263 & 185 & 216 & 129 & 177 & 36 \\
\hline Budget support & 42 & 0 & 0 & 30 & 34 & 34 & 0 \\
\hline Other long-term loans & 186 & 263 & 185 & 185 & 94 & 142 & 36 \\
\hline Scheduled amortization & -14 & -18 & -45 & -48 & -29 & -61 & -29 \\
\hline Other (including short-term capital) & 18 & 102 & -19 & -135 & -118 & -115 & 55 \\
\hline Capital account balance & 743 & 496 & 420 & 1,080 & 966 & 2,353 & 1,406 \\
\hline Errors and omissions & 9 & -42 & -63 & -90 & -47 & -96 & -40 \\
\hline Overall balance & 152 & 266 & 307 & 221 & 119 & 237 & 100 \\
\hline Financing & -152 & -266 & -307 & -221 & -119 & -237 & -100 \\
\hline Change in net foreign assets of ANB (increase -) & -130 & -59 & -74 & -35 & -36 & -124 & -90 \\
\hline Net credit from the Fund & 94 & -52 & -29 & -39 & -20 & -42 & -33 \\
\hline Disbursements/purchases & 110 & 0 & 10 & 10 & 18 & 37 & 0 \\
\hline Repayments/repurchases & -16 & -52 & -39 & -49 & -38 & -79 & -33 \\
\hline Change in gross official reserves (increase -) & -224 & -7 & -45 & 4 & -16 & -82 & -57 \\
\hline Change in other foreign liabilities (increase + ) & 0 & 0 & 0 & 0 & 0 & 0 & 0 \\
\hline Change in arrears (decrease -) & 0 & 15 & 15 & 15 & 8 & 15 & 11 \\
\hline Change in Oil Fund assets (increase-) & -25 & -248 & -221 & -201 & -91 & -128 & -20 \\
\hline Adjustment (Oil Fund required reserves) 1/ & 2 & 25 & -27 & 0 & 0 & 0 & 0 \\
\hline
\end{tabular}

Sources: Azerbaijan National Bank; and Fund staff estimates.

1/ This corrects for the required reserves held at the ANB against the deposits of the Oil Fund prior to 2001, when the Oil Fund's resources were transferred to the ANB from a commercial bank. 
Table 23. Registered Foreign Trade, 1999-2004 1/

(In millions of U.S. dollars)

\begin{tabular}{|c|c|c|c|c|c|c|c|}
\hline & \multirow[t]{2}{*}{1999} & \multirow[t]{2}{*}{2000} & \multirow[t]{2}{*}{2001} & \multirow[t]{2}{*}{2002} & \multicolumn{2}{|c|}{2003} & \multirow{2}{*}{$\frac{2004}{\text { First half }}$} \\
\hline & & & & & First half & Year & \\
\hline Exports & 1,025 & 1,799 & 2,046 & 2,305 & 1,254 & 2,625 & 1,679 \\
\hline Food & 60 & 57 & 55 & 68 & 47 & 127 & 85 \\
\hline Cotton & 22 & 37 & 15 & 23 & 11 & 33 & 18 \\
\hline Oil and oil products & 801 & 1,519 & 1,841 & 2,046 & 1,106 & 2,250 & 1,286 \\
\hline Metals & 25 & 32 & 19 & 22 & 21 & 55 & 48 \\
\hline Chemicals and petrochemicals 2/ & 23 & 36 & 36 & 59 & 24 & 51 & 39 \\
\hline Machinery and equipment & 38 & 34 & 64 & 44 & 16 & 35 & 18 \\
\hline Other & 56 & 84 & 16 & 43 & 30 & 77 & 186 \\
\hline Imports 3 / & 1,036 & 1,172 & 1,465 & 1,823 & 1,164 & 2,723 & 1,553 \\
\hline Food & 210 & 223 & 340 & 351 & 145 & 310 & 219 \\
\hline Natural gas & -- & -- & 180 & 212 & 124 & 215 & 139 \\
\hline Metals & 111 & 123 & 105 & 227 & 237 & 499 & 281 \\
\hline Chemicals and petrochemicals 2 / & 58 & 84 & 64 & 50 & 48 & 110 & 66 \\
\hline Machinery and equipment & 362 & 399 & 347 & 415 & 298 & 738 & 453 \\
\hline Other & 295 & 343 & 429 & 568 & 312 & 851 & 395 \\
\hline
\end{tabular}

Source: Azerbaijan National Bank, Azerbaijan State Statistics Committee and staff estimates.

1/ Import figures for 1999-2000 are based on customs data and are provided by the State Statistics Committee.

Import figures for 1999-2000 differ from Table 22 which is based on central bank data and includes shuttle

trade. Also, the 2000 import figure does not include the import of two Boeings (valued at US\$130 million)

as this import was not reflected in customs data of 2000 (but is included in customs data for October 2001).

The import of these two aircrafts are included in the central bank import data for 2000.

Import figures for 2001 and 2002 are based on data provided by the Azerbaijan National Bank.

2/ Including pharmaceutical products.

3/ Does not include data on shuttle trade for 1999-2000. 
Table 24. Azerbaijan: Balance of Services, Income and Transfers, 1999-2004

(In millions of U.S. dollars)

\begin{tabular}{|c|c|c|c|c|c|c|c|}
\hline & \multirow[t]{2}{*}{1999} & \multirow[t]{2}{*}{2000} & \multirow[t]{2}{*}{2001} & \multirow[t]{2}{*}{2002} & \multicolumn{2}{|c|}{2003} & \multirow{2}{*}{$\begin{array}{c}2004 \\
\text { First half }\end{array}$} \\
\hline & & & & & First half & Year & \\
\hline \multicolumn{8}{|l|}{ Services and Income } \\
\hline Services and Income & -273 & -520 & -707 & $-1,321$ & -951 & $-2,056$ & $-1,498$ \\
\hline Credit & 291 & 316 & 331 & 399 & 238 & 484 & 259 \\
\hline Debit & 564 & 836 & 1,039 & 1,720 & 1,189 & 2,540 & 1,757 \\
\hline Services & -228 & -225 & -375 & -936 & -757 & $-1,614$ & $-1,184$ \\
\hline Credit & 257 & 260 & 290 & 362 & 202 & 431 & 227 \\
\hline Freight & 90 & 88 & 126 & 173 & 69 & 159 & 54 \\
\hline Other transportation & 24 & 31 & 36 & 39 & 24 & 45 & 41 \\
\hline Travel & 81 & 63 & 43 & 51 & 25 & 59 & 29 \\
\hline Other & 62 & 77 & 86 & 99 & 84 & 167 & 103 \\
\hline Debit & 485 & 485 & 665 & 1,298 & 959 & 2,045 & 1,411 \\
\hline Freight & 39 & 101 & 118 & 132 & 70 & 158 & 93 \\
\hline Other transportation & 0 & 43 & 38 & 41 & 9 & 45 & 32 \\
\hline Travel & 139 & 132 & 109 & 105 & 48 & 107 & 59 \\
\hline Government of Azerbaijan (net) & 9 & 9 & 15 & 15 & 10 & 22 & 11 \\
\hline Private services & 80 & 49 & 58 & 146 & 88 & 208 & 159 \\
\hline Oil services & 189 & 154 & 329 & 868 & 734 & 1,505 & 1,057 \\
\hline Income & -45 & -295 & -332 & -386 & -194 & -442 & -314 \\
\hline Credit & 34 & 56 & 41 & 37 & 36 & 53 & 32 \\
\hline Investment income & 34 & 49 & 39 & 35 & 33 & 42 & 27 \\
\hline Other & 0 & 7 & 3 & 2 & 3 & 9 & 5 \\
\hline Debit & 79 & 351 & 374 & 423 & 230 & 495 & 346 \\
\hline Investment income & 47 & 304 & 326 & 384 & 208 & 445 & 298 \\
\hline Interest on public debt & 22 & 26 & 29 & 20 & 10 & 19 & 21 \\
\hline IMF & 12 & 14 & 10 & 5 & 2 & 4 & 1 \\
\hline Other & 11 & 12 & 19 & 15 & 7 & 15 & 19 \\
\hline Profit repatriation of oil consortium & 23 & 278 & 291 & 344 & 189 & 386 & 227 \\
\hline Other investment & 2 & $\ldots$ & $\ldots$ & $\ldots$ & 9 & 40 & 51 \\
\hline Compensation of employees & 32 & 47 & 47 & 39 & 21 & 50 & 49 \\
\hline Transfers (net) & 82 & 73 & 77 & 70 & 62 & 134 & 106 \\
\hline Credit & 135 & 135 & 176 & 228 & 108 & 225 & 138 \\
\hline Debit & 53 & 62 & 100 & 158 & 46 & 91 & 31 \\
\hline Private & 25 & 11 & 13 & 15 & 39 & 77 & 71 \\
\hline Credit & 71 & 69 & 110 & 168 & 81 & 162 & 100 \\
\hline Remittances & 54 & 57 & 104 & 163 & 78 & 152 & 94 \\
\hline Other & 17 & 12 & 6 & 4 & 3 & 10 & 6 \\
\hline Debit & 46 & 59 & 97 & 153 & 42 & 85 & 29 \\
\hline Public & 56 & 62 & 64 & 55 & 23 & 57 & 36 \\
\hline Credit & 63 & 66 & 66 & 60 & 26 & 63 & 38 \\
\hline Current grants for budgetary support & 22 & 17 & 18 & 18 & 0 & 0 & 0 \\
\hline Nonbudgetary current grants & 41 & 48 & 48 & 43 & 26 & 63 & 38 \\
\hline Humanitarian aid & 41 & 48 & 48 & 43 & 17 & 43 & 22 \\
\hline Debit (including subscription fees) & 7 & 3 & 3 & 5 & 4 & 6 & 2 \\
\hline
\end{tabular}

Sources: Azerbaijan National Bank; and Fund staff estimates. 
Table 25. Azerbaijan: Foreign Assistance, 1999-2004

\begin{tabular}{|c|c|c|c|c|c|c|c|}
\hline & \multirow[t]{2}{*}{1999} & \multirow[t]{2}{*}{2000} & \multirow[t]{2}{*}{2001} & \multirow[t]{2}{*}{2002} & \multicolumn{2}{|c|}{2003} & \multirow{2}{*}{$\begin{array}{c}2004 \\
\text { First half }\end{array}$} \\
\hline & & & & & First half & Year & \\
\hline & \multicolumn{6}{|c|}{ (In millions of U.S. dollars) } & \\
\hline \multicolumn{8}{|l|}{ A. Total Aid } \\
\hline Total & 291.6 & 328.5 & 251.3 & 275.9 & 155.2 & 230.6 & 70.4 \\
\hline Multilateral & 277.8 & 312.4 & 235.3 & 261.7 & 146.4 & 212.7 & 60.0 \\
\hline Bilateral & 13.8 & 16.1 & 16.1 & 14.3 & 8.8 & 17.9 & 10.4 \\
\hline Loans & 228.2 & 262.8 & 185.1 & 215.5 & 128.8 & 176.8 & 36.2 \\
\hline Multilateral & 228.2 & 262.8 & 185.1 & 215.5 & 128.8 & 176.8 & 36.2 \\
\hline Bilateral & 0.0 & 0.0 & 0.0 & 0.0 & 0.0 & 0.0 & 0.0 \\
\hline Grants & 63.4 & 65.7 & 66.3 & 60.4 & 26.4 & 53.8 & 34.3 \\
\hline Multilateral & 49.6 & 49.6 & 50.2 & 46.2 & 17.6 & 35.8 & 23.9 \\
\hline Bilateral & 13.8 & 16.1 & 16.1 & 14.3 & 8.8 & 17.9 & 10.4 \\
\hline \multicolumn{8}{|l|}{ B. Loans } \\
\hline Total & 228.2 & 262.8 & 185.1 & 215.5 & 128.8 & 176.8 & 36.2 \\
\hline BOP support (World bank) & 42.0 & 0.0 & 0.0 & 30.3 & 34.4 & 34.4 & 0.0 \\
\hline Projects ( sources of financing) & 186.2 & 262.8 & 185.1 & 185.2 & 94.4 & 142.4 & 36.2 \\
\hline Turkish Eximbank & 1.9 & 0.0 & 0.0 & 0.0 & 0.0 & 0.0 & 0.0 \\
\hline World Bank & 23.9 & 26.7 & 28.5 & 27.1 & 20.6 & 40.3 & 22.4 \\
\hline EBRD & 24.8 & 13.3 & 9.6 & 10.7 & 6.9 & 6.9 & 0.7 \\
\hline KFW & 14.6 & 10.9 & 0.5 & 2.8 & 2.9 & 6.1 & 0.8 \\
\hline IFAD & 1.8 & 1.5 & 2.2 & 0.7 & 1.8 & 2.6 & 0.3 \\
\hline Other & 119.2 & 210.4 & 144.3 & 144.0 & 62.3 & 86.6 & 12.0 \\
\hline \multicolumn{8}{|l|}{ C. Grants } \\
\hline Total & 63.4 & 65.7 & 66.3 & 60.4 & 26.4 & 53.8 & 34.3 \\
\hline BOP support (budgetary) & 22.0 & 17.4 & 18.1 & 17.7 & 0.0 & 0.0 & 11.6 \\
\hline Off budget & 41.4 & 48.3 & 48.2 & 42.8 & 26.4 & 53.8 & 22.7 \\
\hline Total & 63.4 & 65.7 & 66.3 & 60.4 & 26.4 & 53.8 & 34.3 \\
\hline $\mathrm{EU}$ & 22.0 & 17.4 & 18.1 & 17.7 & 0.0 & 0.0 & 3.1 \\
\hline Other & 41.4 & 48.3 & 48.2 & 42.8 & 26.4 & 53.8 & 31.2 \\
\hline
\end{tabular}

Sources: Ministry of Finance; and Fund staff estimates. 
Table 26. Azerbaijan: Direction of Registered Foreign Trade, 1999-2004 1/

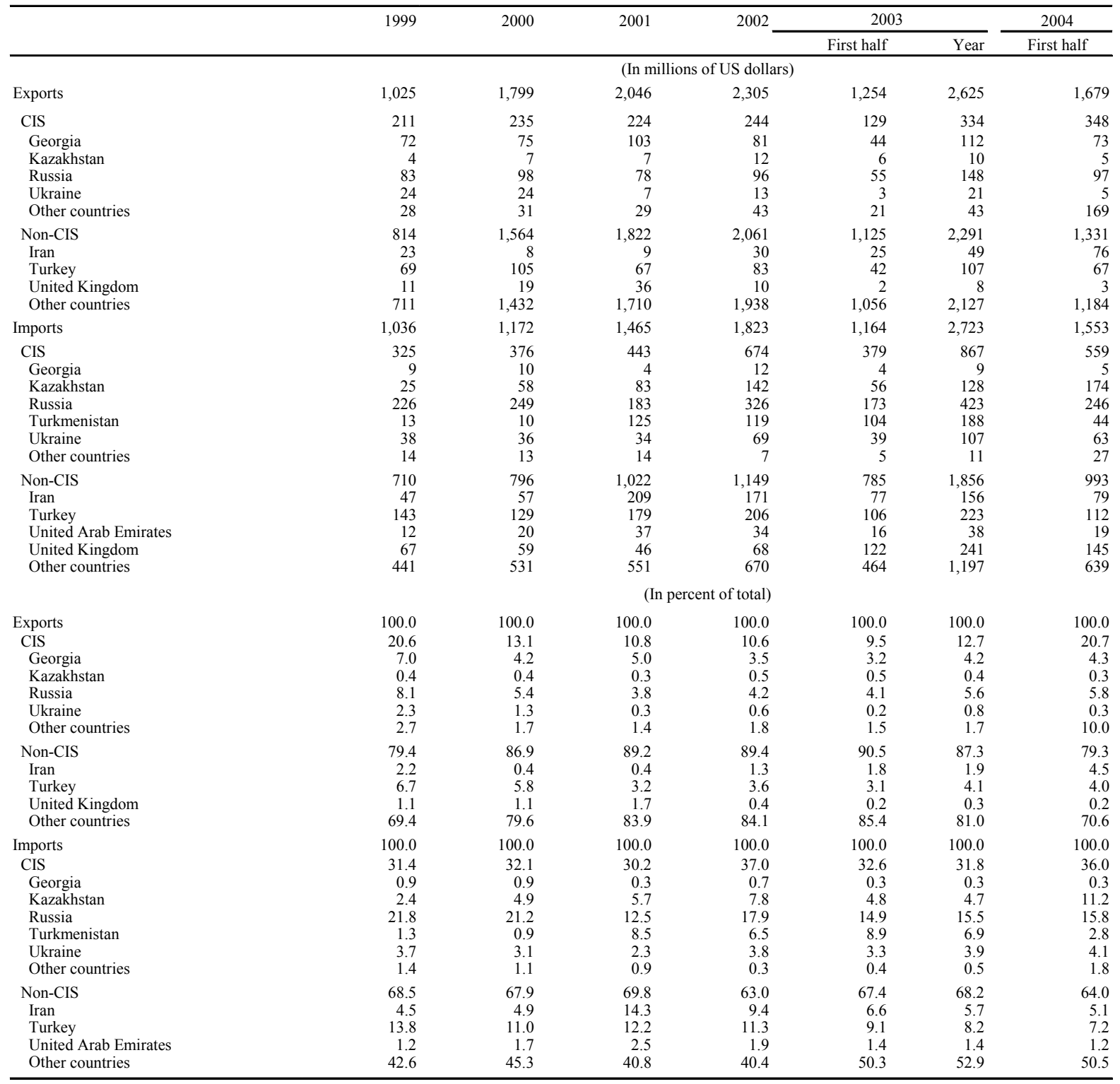

Sources: Azerbaijan National Bank, Azerbaijan State Statistics Committee; and Fund staff estimates.

1/ Import figures for 1999-2000 are based on customs data and are provided by the State Statistics Committee.

Import figures for 1999-2000 differ from Table 22 which is based on central bank data and includes shuttle

trade. Also, the 2000 import figure does not include the import of two Boeings (valued at US\$130 million)

as this import was not reflected in customs data of 2000 (but is included in customs data for October 2001).

The import of these two aircrafts are included in the central bank import data for 2000.

Import figures for 2001 and 2002 are based on data provided by the Azerbaijan National Bank. 
Table 27. Energy-Related Subsidies and Consolidated Government Operations, 2001-04 1/2/

\begin{tabular}{|c|c|c|c|c|c|c|c|c|}
\hline & \multicolumn{2}{|c|}{2001} & \multicolumn{2}{|c|}{2002} & \multicolumn{2}{|r|}{2003} & \multicolumn{2}{|c|}{$20043 /$} \\
\hline & cash basis & $\begin{array}{c}\text { Including } \\
\text { subsidies 3/ }\end{array}$ & cash basis & $\begin{array}{c}\text { Including } \\
\text { subsidies 3/ }\end{array}$ & cash bas & $\begin{array}{c}\text { Including } \\
\text { subsidies 3/ }\end{array}$ & cash basis & $\begin{array}{c}\text { Including } \\
\text { subsidies 3/ }\end{array}$ \\
\hline & \multicolumn{8}{|c|}{ (In billions of manat) } \\
\hline Total revenue and grants & 4,961 & 6,646 & 6,673 & 8,276 & 7,611 & 9,506 & 4,044 & 5,290 \\
\hline Tax revenue & 3,891 & 5,576 & 4,575 & 6,178 & 5494 & 7389 & 2,961 & 4,207 \\
\hline Nontax revenue & 1,043 & 1,043 & 2,041 & 2,041 & 2,088 & 2,088 & 1,083 & 1,083 \\
\hline Grants & 28 & 28 & 57 & 57 & 28 & 28 & 0 & 0 \\
\hline Total expenditure & 4,960 & 7,041 & 6,785 & 8,388 & 8,219 & 10,114 & 3,740 & 4,986 \\
\hline Current expenditure & 4,412 & 6,097 & 5,086 & 6,689 & 6,077 & 7,972 & 3,119 & 4,365 \\
\hline Investment and net lending & 547 & 943 & 1,699 & 1,699 & 2,142 & 2,142 & 621 & 621 \\
\hline Consolidated government deficit, cash basis & -115 & -115 & -144 & -144 & -425 & -425 & 450 & 450 \\
\hline \multirow[t]{2}{*}{ Statistical discrepancy } & -116 & -116 & 32 & 32 & -184 & -184 & -146 & -146 \\
\hline & \multicolumn{8}{|c|}{ (In percent of GDP) } \\
\hline Total revenue and grants & 18.7 & 25.0 & 22.0 & 27.3 & 21.7 & 27.1 & 21.8 & 28.6 \\
\hline Tax revenue & 14.6 & 21.0 & 15.1 & 20.4 & 15.7 & 21.1 & 16.0 & 22.7 \\
\hline Nontax revenue & 3.9 & 3.9 & 6.7 & 6.7 & 6.0 & 6.0 & 5.8 & 5.8 \\
\hline Grants & 0.1 & 0.1 & 0.2 & 0.2 & 0.1 & 0.1 & 0.0 & 0.0 \\
\hline Total expenditure & 18.7 & 26.5 & 22.4 & 27.7 & 23.4 & 28.9 & 20.2 & 26.9 \\
\hline Current expenditure & 16.6 & 22.9 & 16.8 & 22.1 & 17.3 & 22.7 & 16.8 & 23.6 \\
\hline Investment and net lending & 2.1 & 3.5 & 5.6 & 5.6 & 6.1 & 6.1 & 3.4 & 3.4 \\
\hline Consolidated government deficit, cash basis & -0.4 & -0.4 & -0.5 & -0.5 & -1.2 & -1.2 & 2.4 & 2.4 \\
\hline Statistical discrepancy & -0.4 & -0.4 & 0.1 & 0.1 & -0.5 & -0.5 & -0.8 & -0.8 \\
\hline \multicolumn{9}{|l|}{ Memorandum item: } \\
\hline Nominal GDP (In billions of manat) & 26,578 & 26,578 & 30,312 & 30,312 & 35,054 & 35,054 & 18,520 & 18,520 \\
\hline
\end{tabular}


Table 28. Azerbaijan: Energy Balance, 1999-2003

\begin{tabular}{|c|c|c|c|c|c|}
\hline & 1999 & 2000 & 2001 & 2002 & 2003 \\
\hline & \multicolumn{5}{|c|}{ (In millions of barrels) } \\
\hline \multicolumn{6}{|l|}{ Oil and oil products } \\
\hline Production & 100.8 & 102.2 & 147.6 & 150.8 & 152.9 \\
\hline Net volume of trade & 47.4 & 55.0 & 77.6 & 85.6 & 79.8 \\
\hline Exports & 49.1 & 55.4 & 77.6 & 85.6 & 79.8 \\
\hline of which: crude oil & 31.2 & 40.6 & 61.9 & 64.9 & 65.8 \\
\hline Imports & -1.7 & -0.4 & 0.0 & 0.0 & 0.0 \\
\hline Domestic consumption & 53.4 & 47.2 & 70.0 & 65.2 & 73.1 \\
\hline Changes in stocks & -0.6 & -1.0 & -1.0 & 1.4 & 0.1 \\
\hline Natural gas & \multicolumn{5}{|c|}{ (In billions of cubic meters) } \\
\hline Production (excluding losses) 1/ & 6.0 & 5.6 & 5.5 & 5.1 & 5.1 \\
\hline Net volume of trade & 0.0 & 0.0 & -3.3 & -3.9 & -4.1 \\
\hline Exports & 0.0 & 0.0 & 0.0 & 0.0 & 0.0 \\
\hline Imports & 0.0 & 0.0 & -3.3 & -3.9 & -4.1 \\
\hline Domestic consumption & 6.0 & 5.6 & 8.8 & 9.0 & 9.2 \\
\hline Changes in stocks & 0.0 & 0.0 & 0.0 & 0.0 & 0.0 \\
\hline
\end{tabular}

Sources: Ministry of Economic Development, the State Statistics Committee; and Fund staff estimates.

1/ Production excludes vented gas. 
Table 29. Azerbaijan: Collection Rates of Azerigas for Gas Supplied to Various Consumers, 1999-2004

\begin{tabular}{|c|c|c|c|c|}
\hline & $\begin{array}{r}\text { Quantity } \\
\text { (million cbm) }\end{array}$ & $\begin{array}{r}\text { Value } \\
\text { (in mln manat) } \\
\end{array}$ & $\begin{array}{c}\text { Actual Payments } \\
\text { Received (in mln manat) }\end{array}$ & $\begin{array}{r}\text { Collection Rate } \\
\text { (In percent) } \\
\end{array}$ \\
\hline 1999 Total supplied gas & 4,273 & 517,080 & 203,809 & 39.4 \\
\hline Population & 1,638 & 58,230 & 26,200 & 45.0 \\
\hline Budgetary organizations 1/ & 820 & 88,558 & 86,780 & 98.0 \\
\hline Industry & 415 & 109,495 & 58,709 & 53.6 \\
\hline JSC "Azerenergy" & 1,268 & 250,985 & 31,600 & 12.6 \\
\hline SOCAR & 134 & 9,812 & 520 & 5.3 \\
\hline 2000 Total supplied gas & 4,165 & 431,581 & 97,781 & 22.7 \\
\hline Population & 1,702 & 39,819 & 18,732 & 47.0 \\
\hline Budgetary organizations $1 /$ & 741 & 42,958 & 12,425 & 28.9 \\
\hline Industry & 447 & 104,758 & 61,346 & 58.6 \\
\hline JSC "Azerenergy" & 1,160 & 230,330 & 4,676 & 2.0 \\
\hline SOCAR & 116 & 13,716 & 603 & 4.4 \\
\hline 2001 Total supplied gas & 3,906 & 399,798 & 120,283 & 30.1 \\
\hline Population & 2,023 & 75,827 & 24,112 & 31.8 \\
\hline Budgetary organizations 1/ & 516 & 53,978 & 13,030 & 24.1 \\
\hline Industry & 491 & 106,147 & 21,193 & 20.0 \\
\hline JSC "Azerenergy" & 826 & 158,885 & 60,588 & 38.1 \\
\hline SOCAR & 51 & 4,962 & 1,360 & 27.4 \\
\hline 2002 Total supplied gas & 3,784 & 277,272 & 134,929 & 48.7 \\
\hline Population & 2,394 & 85,169 & 43,219 & 50.7 \\
\hline Budgetary organizations $1 /$ & 520 & 53,194 & 16,841 & 31.7 \\
\hline Industry & 597 & 97,404 & 69,839 & 71.7 \\
\hline JSC "Azerenergy" & 91 & 17,839 & 3,904 & 21.9 \\
\hline SOCAR & 183 & 23,667 & 1,126 & 4.8 \\
\hline 2003 Total supplied gas $1 /$ & $4,167.1$ & $337,953.1$ & $180,605.8$ & 53.4 \\
\hline Population & $2,696.3$ & $95,879.7$ & $45,236.0$ & 47.2 \\
\hline Budgetary organizations $2 /$ & 460.7 & $48,987.5$ & $13,140.8$ & 26.8 \\
\hline Industry & 796.3 & $165,716.2$ & $105,949.4$ & 63.9 \\
\hline SOCAR & 213.8 & $27,369.7$ & $16,279.6$ & 59.5 \\
\hline 2004 Total supplied gas $3 /$ & $2,263.9$ & $186,781.5$ & $121,898.9$ & 65.3 \\
\hline Population & $1,481.6$ & $52,684.1$ & $29,641.1$ & 56.3 \\
\hline Budgetary organizations $1 /$ & 249.9 & $29,766.0$ & $5,569.6$ & 18.7 \\
\hline Industry & 453.6 & $93,581.7$ & $86,270.2$ & 92.2 \\
\hline SOCAR & 78.8 & $10,749.7$ & 418.0 & 3.9 \\
\hline
\end{tabular}

Source: Azerigas.

1/ Includes organizations that are not part of the general government. Since 2003, the general government has been paying in full its energy fees.

2/ Starting from 2003 Azerenegy only uses natural gas imported by SOCAR.

3/ First half, preliminary estimate. 
Table 30. Azerbaijan: Collection Rates of Azerenergy for Electricity Supplied to Various Consumers, 1999-2004

\begin{tabular}{|c|c|c|c|c|}
\hline & $\begin{array}{r}\text { Quantity } \\
\text { (million kwh) } \\
\end{array}$ & $\begin{array}{r}\text { Value } \\
\text { (in mln Manat) } \\
\end{array}$ & $\begin{array}{r}\text { Actual Payments } \\
\text { Received (in mln Manat) } \\
\end{array}$ & $\begin{array}{r}\text { Collection Rate } \\
\text { (In percent) } \\
\end{array}$ \\
\hline 1999 Total supplied electricity & $15,002.1$ & $1,612.1$ & 554.7 & 34.4 \\
\hline Population & $9,229.3$ & 461.5 & 97.5 & 21.1 \\
\hline Budgetary organizations 1 / & $1,006.8$ & 318.4 & 77.9 & 24.5 \\
\hline State Owned Enterprises & 783.2 & 150.3 & 21.1 & 14.0 \\
\hline Industry & $3,202.8$ & 614.9 & 350.2 & 57.0 \\
\hline Other consumers & 780.0 & 67.0 & 8.1 & 12.0 \\
\hline 2000 Total supplied electricity & $15,607.5$ & $1,533.3$ & 244.7 & 16.0 \\
\hline Population & $9,963.3$ & 518.1 & 68.4 & 13.2 \\
\hline Budgetary organizations 1 / & $1,039.7$ & 172.3 & 61.5 & 35.7 \\
\hline State Owned Enterprises & 787.7 & 203.0 & 12.7 & 6.3 \\
\hline Industry & $2,856.8$ & 497.9 & 87.2 & 17.5 \\
\hline Other consumers & 960.0 & 142.0 & 14.9 & 10.5 \\
\hline 2001 Total supplied electricity $2 /$ & $16,161.7$ & $1,365.7$ & 474.0 & 34.7 \\
\hline Population & $10,232.0$ & 532.0 & 98.4 & 18.5 \\
\hline Budgetary organizations $1 /$ & $1,084.7$ & 163.4 & 54.8 & 33.5 \\
\hline State Owned Enterprises & 784.6 & 213.7 & 35.3 & 16.5 \\
\hline Industry & $2,976.4$ & 456.6 & 285.5 & 62.5 \\
\hline Other consumers 3 / & $1,084.0$ & $\ldots$ & $\ldots$ & ... \\
\hline 2002 Total supplied electricity 2 / & $18,031.2$ & $1,737.7$ & 598.9 & 34.5 \\
\hline Population & $10,540.3$ & $1,007.4$ & 139.2 & 13.8 \\
\hline Budgetary organizations 1 / & 977.5 & 149.9 & 110.2 & 73.5 \\
\hline State Owned Enterprises & 641.1 & 135.4 & 107.8 & 79.6 \\
\hline Industry & $2,942.7$ & 445.0 & 241.7 & 54.3 \\
\hline Other consumers 3 / & $2,929.6$ & $\ldots$ & $\ldots$ & $\ldots$ \\
\hline 2003 Total supplied electricity $2 /$ & $17,181.4$ & $1,944.7$ & 877.2 & 45.1 \\
\hline Population & 12074.5 & 1158.6 & 242.6 & 20.9 \\
\hline Budgetary organizations 1/ & 879.6 & 134.7 & 112.1 & 83.2 \\
\hline State Owned Enterprises & $\ldots$ & $\ldots$ & $\ldots$ & $\ldots$ \\
\hline Industry & 2955.8 & 419.0 & 309.9 & 74.0 \\
\hline Other consumers & 1271.5 & 232.4 & 212.5 & 91.5 \\
\hline 2004 Total supplied electricity $2 / 4$ / & $9,289.4$ & $1,055.5$ & 499.1 & 47.3 \\
\hline Population & 6480.9 & 622.0 & 144.5 & 23.2 \\
\hline Budgetary organizations 1 / & 469.7 & 72.0 & 47.7 & 66.3 \\
\hline State Owned Enterprises & $\ldots$ & $\ldots$ & $\ldots$ & $\ldots$ \\
\hline Industry & 1616.5 & 223.9 & 190.2 & 84.9 \\
\hline Other consumers & 722.4 & 137.7 & 116.8 & 84.8 \\
\hline
\end{tabular}

Source: Azerenergy.

1/ Includes organizations that are not part of the general government. Since 2003, the general government has been paying in full its energy fees.

2/ Starting from the second half of 2001, Azerenergy no longer distributes electricity directly to end-users. Instead,

it supplies electricity to four distribution network companies, which in turn distribute electricity to end users.

3/ Data are not available.

4/ First half, preliminary estimate. 